\title{
Measurement of neutral strange particle production in the underlying event in proton-proton collisions at $\sqrt{s}=7 \mathrm{TeV}$
}

\author{
S. Chatrchyan et al.* \\ (CMS Collaboration)
}

(Received 26 May 2013; published 3 September 2013)

\begin{abstract}
Measurements are presented of the production of primary $K_{S}^{0}$ and $\Lambda$ particles in proton-proton collisions at $\sqrt{s}=7 \mathrm{TeV}$ in the region transverse to the leading charged-particle jet in each event. The average multiplicity and average scalar transverse momentum sum of $K_{S}^{0}$ and $\Lambda$ particles measured at pseudorapidities $|\eta|<2$ rise with increasing charged-particle jet $p_{\mathrm{T}}$ in the range $1-10 \mathrm{GeV} / c$ and saturate in the region $10-50 \mathrm{GeV} / c$. The rise and saturation of the strange-particle yields and transverse momentum sums in the underlying event are similar to those observed for inclusive charged particles, which confirms the impact-parameter picture of multiple parton interactions. The results are compared to recent tunes of the PYTHIA Monte Carlo event generator. The PYTHIA simulations underestimate the data by $15 \%-30 \%$ for $K_{S}^{0}$ mesons and by about $50 \%$ for $\Lambda$ baryons, a deficit similar to that observed for the inclusive strange-particle production in non-single-diffractive proton-proton collisions. The constant strange- to charged-particle activity ratios with respect to the leading jet $p_{\mathrm{T}}$ and similar trends for mesons and baryons indicate that the multiparton-interaction dynamics is decoupled from parton hadronization, which occurs at a later stage.
\end{abstract}

DOI: 10.1103/PhysRevD.88.052001

PACS numbers: 12.38.Aw, 13.85.Ni

\section{INTRODUCTION}

This paper describes a measurement of the production of primary $K_{S}^{0}$ mesons, and $\Lambda$ and $\bar{\Lambda}$ baryons in the underlying event in proton-proton $(p p)$ collisions at a center-ofmass energy of $7 \mathrm{TeV}$ with the Compact Muon Solenoid (CMS) detector at the Large Hadron Collider (LHC).

In the presence of a hard process, characterized by particles or clusters of particles with large transverse momentum $p_{\mathrm{T}}$ with respect to the beam direction, the final state of hadron-hadron interactions can be described as the superposition of several contributions: the partonic hard scattering, initial- and final-state radiation, additional "multiple partonic interactions" (MPI), and "beam-beam remnants" (BBR) interactions. The products of initial- and final-state radiation, MPI and BBR, form the "underlying event" (UE).

In this paper, the UE properties are analyzed with reference to the direction of the highest- $p_{\mathrm{T}}$ jet reconstructed from charged primary particles (leading charged-particle jet). This leading jet is expected to reflect the direction of the parton produced with the highest transverse momentum in the hard interaction. Three distinct topological regions in the hadronic final state are defined in terms of the azimuthal angle $\Delta \phi$ between the directions of the leading jet and that of any particle in the event. Particle production in the "toward" region, $|\Delta \phi|<60^{\circ}$, and in the "away" region,

*Full author list given at the end of the article.

Published by the American Physical Society under the terms of the Creative Commons Attribution 3.0 License. Further distribution of this work must maintain attribution to the author(s) and the published article's title, journal citation, and DOI.
$|\Delta \phi|>120^{\circ}$, is expected to be dominated by the hard parton-parton scattering. The UE structure can be best studied in the "transverse" region, $60^{\circ}<|\Delta \phi|<120^{\circ}[1,2]$.

Studies of the UE activity in charged primary particles in proton-proton collisions at different center-of-mass energies have been published by the ATLAS [3] and CMS $[1,4,5]$ collaborations. Observables such as the average multiplicity of charged primary particles per event, hereafter referred to as "average rate," and the average scalar sum of primary particle $p_{\mathrm{T}}$ per event, hereafter referred to as "average $p_{\mathrm{T}}$ sum," have been measured in the transverse region. These quantities exhibit a steep rise with increasing charged-particle jet $p_{\mathrm{T}}$ up to a value that depends on the proton-proton center-of-mass energy (around $10 \mathrm{GeV} / c$ for $p p$ collisions at $7 \mathrm{TeV}$ ), followed by a slow rise. Within the MPI framework, a hard jet is likely to be produced in collisions with a small impact parameter between the colliding protons, consequently resulting in large MPI activity $[6,7]$. The MPI activity saturates at values of the hard scale typical of central collisions.

The present analysis considers identified neutral strange particles $\left(K_{S}^{0}, \Lambda\right.$, and $\left.\bar{\Lambda}\right)$ as additional probes to study the underlying event. Unless stated otherwise, $\Lambda$ and $\bar{\Lambda}$ baryon data are merged and referred to as $\Lambda$ baryon data. The production of primary $K_{S}^{0}$ and $\Lambda$ particles in the transverse region at $\sqrt{s}=7 \mathrm{TeV}$ is studied as a function of the scale of the hard process. Fully corrected average rates and $p_{\mathrm{T}}$ sums of primary $K_{S}^{0}$ mesons and $\Lambda$ baryons, as well as ratios to the charged primary-particle rates and $p_{\mathrm{T}}$ sums, are compared to simulations. This analysis complements the studies of strangeness production in minimum-bias events at $\sqrt{s}=7 \mathrm{TeV}$ published by the ALICE $[8,9]$, 
ATLAS [10], and CMS [11] collaborations. Comparisons of nonsingle diffractive data [11] with predictions made with the PYTHIA 6 [12] and PYTHIA 8 [13] Monte Carlo event generators have shown that the latter largely underestimate the data, e.g., by $30 \%$ for $K_{S}^{0}$ production and $50 \%$ for $\Lambda$ production at $\sqrt{s}=7 \mathrm{TeV}$ for PYTHIA 6 tune D6T $[2,14]$, with little improvement for more recent tunes.

The simulations are performed with versions of PYTHIA that include MPI. The most recent versions have been tuned to reproduce the UE activity observed with primary charged particles at the $\mathrm{LHC}$ at $0.9 \mathrm{TeV}$ and $7 \mathrm{TeV}$ centerof-mass energies. The parameters describing strangeness production, however, have not been tuned to LHC data yet. All Monte Carlo samples used in this paper have been generated with the default values of these parameters.

Recent literature [15-17] discussing the tuning of the strangeness suppression parameters in commonly available generators is limited. A tuning of the PYTHIA 6 parameters to LEP, SLAC Linear Collider, and Tevatron data performed with the PROFESSOR program [15] produced bestfit parameters in disagreement with the current PYTHIA default parameters. The resulting predicted strange meson and baryon production rates given in the Appendix of Ref. [15], however, do not agree well with the data used for the tuning. Other attempts to describe strange-particle production in $p p$ collisions are discussed in Refs. [16,17]. The present paper focuses on the comparison with PYTHIA.

The outline of this paper is the following. In Sec. II, the experimental conditions are described, along with the data sets, the simulation, and the analysis technique. In Sec. III, the systematic uncertainties are summarized. The results are discussed in Sec. IV, and conclusions are drawn in Sec. V.

\section{EXPERIMENTAL SETUP, DATA SETS, AND DATA ANALYSIS}

The central feature of CMS is a superconducting solenoid of $6 \mathrm{~m}$ internal diameter. Within the superconducting solenoid volume are a silicon pixel and strip tracker, a lead tungstate crystal electromagnetic calorimeter, and a brass and scintillator hadron calorimeter. Muons are measured in gas-ionization detectors embedded in the flux-return yoke. Extensive forward calorimetry complements the coverage provided by the barrel and endcap detectors. CMS uses a right-handed coordinate system, with the origin at the nominal interaction point, the $x$ axis pointing to the center of the LHC, the $y$ axis pointing up (perpendicular to the LHC plane), and the $z$ axis along the anticlockwise-beam direction. The polar angle $\theta$ is measured from the positive $z$ axis, and the azimuthal angle $\phi$ is measured in the $x-y$ plane. The tracker measures charged particles within the pseudorapidity range $|\eta|<2.5$, where $\eta=$ $-\ln (\tan (\theta / 2))$. It consists of 1440 silicon pixel and 15148 silicon strip detector modules and is located in the $3.8 \mathrm{~T}$ field of the superconducting solenoid. For the charged particles of interest in this analysis, the transverse momentum resolution is relatively constant with $p_{\mathrm{T}}$, varying from $0.7 \%$ at $\eta=0$ to $2 \%$ at $|\eta|=2$. The transverse and longitudinal impact-parameter resolutions, $\sigma_{d_{0}}$ and $\sigma_{d_{z}}$, respectively, depend on $p_{\mathrm{T}}$ and on $\eta$, ranging from $\sigma_{d_{0}}=400 \mu \mathrm{m}$ and $\sigma_{d_{z}}=1000 \mu \mathrm{m}$ at $p_{\mathrm{T}}=0.3 \mathrm{GeV} / c$ and $|\eta|>1.4$ to $\sigma_{d_{0}}=10 \mu \mathrm{m}$ and $\sigma_{d_{z}}=30 \mu \mathrm{m}$ at $p_{\mathrm{T}}=$ $100 \mathrm{GeV} / c$ and $|\eta|<0.9$. A more detailed description of the CMS detector can be found in Ref. [18].

\section{A. Event selection, data sets, and Monte Carlo simulation}

The event selection is identical to the one described in [1], unless explicitly stated otherwise. Minimum-bias events were triggered by requiring coincident signals in beam scintillator counters located on both sides of the experiment and covering the pseudorapidity range $3.23<$ $|\eta|<4.65$, and in the beam pickup devices [18]. Events were then recorded with a prescaled trigger requiring the presence of at least one track segment in the pixel detector with $p_{\mathrm{T}}>200 \mathrm{MeV} / c$. The trigger conditions are applied to both data and simulated samples. The trigger efficiency for the events selected in the analysis is close to $100 \%$, and no bias from the trigger selection is found.

The data used in this analysis were collected in early 2010 when pileup (multiple $p p$ collisions per proton bunch crossing) was very low. Selected events are required to contain a single reconstructed primary vertex, a condition that rejects about $1 \%$ of the events satisfying all the other selection criteria. The primary vertex is fit with an adaptive algorithm [19] and must have at least four tracks, a transverse distance to the beam line smaller than $2 \mathrm{~cm}$, and a $z$ coordinate within $10 \mathrm{~cm}$ of the nominal interaction point.

Events are required to contain a track jet with reconstructed $p_{\mathrm{T}}>1 \mathrm{GeV} / c$ and $|\eta|<2$. Track jets are reconstructed from the tracks of charged particles, with the anti- $k_{\mathrm{T}}$ algorithm [20,21] and a clustering radius $\Delta R=$ 0.5 , where $\Delta R=\sqrt{(\Delta \eta)^{2}+(\Delta \phi)^{2}}$. The tracks are required to be well reconstructed, to have $p_{\mathrm{T}}>500 \mathrm{MeV} / c,|\eta|<$ 2.5 , and to be consistent with originating from the primary vertex. More details on the track selection can be found in [1]. The reconstructed track jet $p_{\mathrm{T}}$ is the magnitude of the vector sum of the transverse momenta of the tracks in the jet. The leading track jet $p_{\mathrm{T}}$ is corrected for detector response (track finding efficiency and $p_{\mathrm{T}}$ measurement) with detailed simulations based on GEANT4 [22], which have been extensively validated with data [23-25]. This correction is approximately independent of the track jet $p_{\mathrm{T}}$ and $\eta$, and its average value is 1.01 . The leading corrected track jet is referred to as the leading charged-particle jet.

The PYTHIA versions we consider all include MPI. The tunes used are the PYTHIA 6 D6T tune $[2,14]$ and the PYTHIA 8 tune 1 [13], which have not been tuned to the LHC data, and the PYTHIA $6 \mathrm{Z1}$ [26] and $\mathrm{Z2}^{*}$ tunes. The two latter 
PYTHIA 6 tunes, as well as PYTHIA 8 , include $p_{\mathrm{T}}$ ordering of the parton showers, and a new model [27] where MPI are interleaved with parton showering. PYTHIA 8 includes hard diffraction in addition to the new MPI model. The parton distribution functions used for PYTHIA 6 D6T and PYTHIA 8 tune 1 are the CTEQ6L1 and CTEQ5L sets, respectively. The Z1 tune uses the CTEQ5L parton distribution set, whereas $\mathrm{Z2}^{*}$ is updated to CTEQ6L1 [28] and retuned to the underlying event activity at $7 \mathrm{TeV}$ from Ref. [1] with the PROFESSOR tool [15]. The simulated data are generated with PYTHIA 6 version 6.422 for tunes D6T and Z1, version 6.424 for tune $\mathrm{Z2}^{*}$, and version 8.135 for PYTHIA 8 tune 1 .

Simulated primary stable charged particles with a proper lifetime $c \tau>1 \mathrm{~cm}$ are clustered into jets with the anti- $k_{\mathrm{T}}$ algorithm $(\Delta R=0.5)$. The average rates and scalar $p_{\mathrm{T}}$ sums of simulated primary $K_{S}^{0}$ and $\Lambda$ particles are computed within the transverse region of the leading simulated charged-particle jet.

A data sample of $11 \times 10^{6}$ events with at least one charged-particle jet with $p_{\mathrm{T}}>1 \mathrm{GeV} / c$ and $|\eta|<2$ is analyzed. The corresponding numbers of simulated events are $22 \times 10^{6}$ for PYTHIA 6 D6T and $5 \times 10^{6}$ for PYTHIA 6 $\mathrm{Z} 1, \mathrm{Z2}^{*}$ and PYTHIA 8 tune 1 . Corrections for detector effects and background are estimated with the PYTHIA 6 D6T sample, while the modeling of the underlying event is studied with all the tunes mentioned.

The reconstruction of the leading charged-particle jet results in a bias in the measured average rates and $p_{\mathrm{T}}$ sums in the transverse region. The value of this bias ranges from $+5 \%$ to $+10 \%$ for charged-particle jet $p_{\mathrm{T}}$ below $10 \mathrm{GeV} / c$, and is consistent with zero for larger $p_{\mathrm{T}}$ values. It is caused by events in which the leading jet formed by primary charged particles is not reconstructed as the leading charged-particle jet because of tracking inefficiencies, and a subleading jet is thus reconstructed as the leading jet. This results in a reconstructed transverse region shifted in $\phi$. The correction for this bias is obtained from the detailed Monte Carlo simulations of the detector response described above.

The primary vertex selection causes a small overestimate of the UE strangeness activity at low charged-particle jet $p_{\mathrm{T}}$, at most $5 \%$ for charged-particle jet $p_{\mathrm{T}}=1 \mathrm{GeV} / c$. This is because the requirement that at least four tracks be associated to the primary vertex enriches the sample in events with higher UE activity when the charged-particle jets have very low multiplicity. This bias is corrected by means of detailed simulations as described in Sec. III.

\section{B. Selection of primary $V^{\mathbf{0}}$ candidates and analysis strategy}

The neutral strange particles $K_{S}^{0}, \Lambda$, and $\bar{\Lambda}$, hereafter generically called $V^{0} \mathrm{~s}$, are identified by means of their characteristic decay topology: a flight distance of several centimeters before decay, two tracks of opposite charge emerging from a secondary vertex, and an invariant mass consistent with that of a $K_{S}^{0}$ meson or a $\Lambda$ baryon. The $V^{0}$ momentum vector is further required to be collinear with the vector joining the primary and secondary vertices, in order to select primary particles.

The $V^{0}$ candidates are reconstructed by the standard CMS offline event reconstruction program [25]. Pairs of oppositely charged tracks with at least 3 hits in the CMS tracker and with a nonzero transverse impact parameter with respect to the beam line are selected (the transverse impact parameter divided by its uncertainty is required to be larger than 1.5). Pairs of tracks with a distance of closest approach to each other smaller than $1 \mathrm{~cm}$ are fit to a common secondary vertex, and those with a vertex fit $\chi^{2}$ smaller than 7 and a significant distance between the beam line and the secondary vertex (transverse flight distance divided by its uncertainty larger than 8) are retained.

Well-reconstructed $V^{0}$ candidates are selected by applying cuts on the pseudorapidity and transverse momentum of the decay tracks $\left(|\eta|<2.5, p_{\mathrm{T}}>300 \mathrm{MeV} / c\right)$, of the $V^{0}$ candidate $\left(|\eta|<2 ; p_{\mathrm{T}}>600 \mathrm{MeV} / c\right.$ for $K_{S}^{0}$ mesons, $p_{\mathrm{T}}>1.5 \mathrm{GeV} / c$ for $\Lambda$ baryons), and on the $V^{0}$ transverse flight distance ( $>1 \mathrm{~cm}$ from the beam line). A kinematic fit is then performed on the candidates to further purify the sample of primary strange particles. The fit includes a secondary vertex constraint, a mass constraint, as well as the constraint that the $V^{0}$ momentum points away from the primary vertex. All three hypotheses $\left(K_{S}^{0} \rightarrow \pi^{+} \pi^{-}\right.$, $\Lambda \rightarrow p \pi^{-}$, and $\bar{\Lambda} \rightarrow \bar{p} \pi^{+}$) are tested for each candidate and the most probable hypothesis is considered. Candidates with a kinematic-fit probability larger than $5 \%$ are retained.

Since simulations enter in the determination of the $V^{0}$ selection efficiency and purity, a good description of the distributions of the kinematic-fit input variables is important. The distributions of the invariant mass of the $V^{0}$ candidates for the most probable particle-type hypothesis are shown in Fig. 1, together with the distributions of the invariant-mass pull. The invariant-mass pull is the difference between the reconstructed mass and the accepted $V^{0}$ mass value [29], divided by the uncertainty on the reconstructed mass calculated from the decay track parameter uncertainties. The signal and background fractions are shown as predicted by PYTHIA 6 D6T. The backgrounds in the $K_{S}^{0}$ sample are mostly misidentified $\Lambda$ baryons. Backgrounds in the $\Lambda$ sample are mostly nonprimary $\Lambda$ baryons from cascade decays of $\Xi$ and $\Omega$ baryons, plus contributions from misidentified $K_{S}^{0}$ mesons and converted photons. In general, the simulation agrees with the data. As an example, the average mass values for $K_{S}^{0}$ mesons $(\Lambda$ baryons) are $0.4981 \mathrm{GeV} / c^{2}\left(1.116 \mathrm{GeV} / c^{2}\right)$ in the simulation and $0.4977 \mathrm{GeV} / c^{2}\left(1.116 \mathrm{GeV} / c^{2}\right)$ in the data; the corresponding rms values for the mass pull distributions are $1.17(0.512)$ in the simulation and $1.23(0.531)$ in the data. For $K_{S}^{0}$ candidates, the data show larger tails than the simulation at mass pull values below $(-2)$. The presence of a 

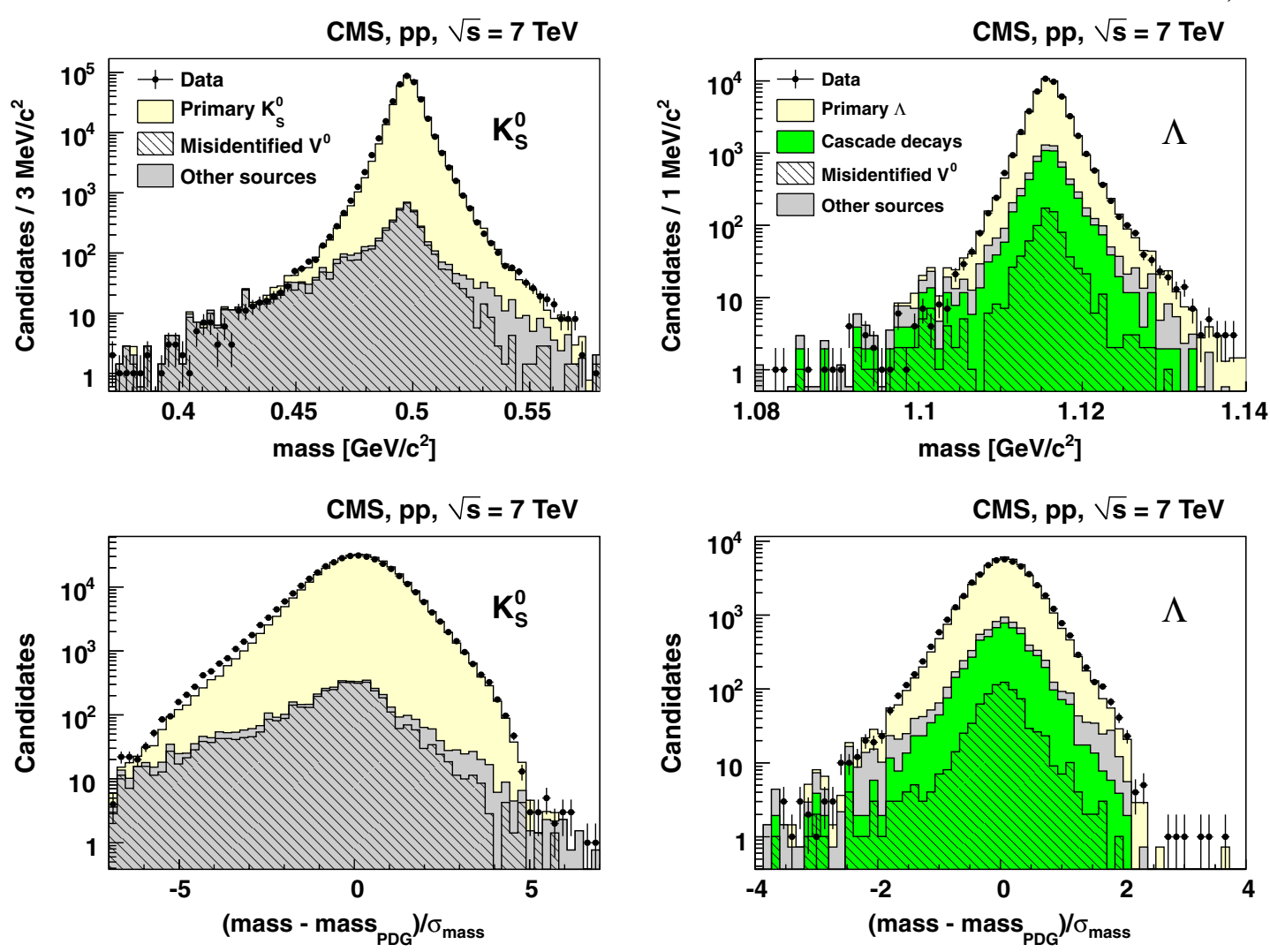

FIG. 1 (color online). Distributions of invariant mass and invariant-mass pull for the most probable particle-type hypothesis

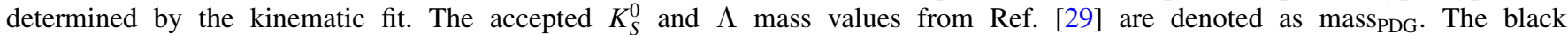
points indicate the data. The histograms show the backgrounds (hatched: misidentified $V^{0}$; green: nonprimary $\Lambda$ from $\Xi$ and $\Omega$ cascade decays; grey: other sources) and the signal (yellow) as predicted by PYTHIA 6 D6T. The PYTHIA prediction is normalized to the data.

similar tail in the component shown as the hatched histogram of the simulated distribution indicates that this excess is due to a larger contribution from misidentified baryons in the data compared to the simulation. This is accounted for in the background estimation as described below.

The pointing requirement constrains the signed impact parameter $d_{\text {ip }}$ of the $V^{0}$ with respect to the primary vertex. This variable is defined as the distance of closest approach of the $V^{0}$ trajectory to the primary vertex, and its sign is that of the scalar product of the $V^{0}$ momentum and the vector pointing from the primary vertex to the point of closest approach. The distributions of the signed impact parameter are shown in Fig. 2 together with the distributions of the corresponding pull, defined as $d_{\mathrm{ip}}$ divided by its uncertainty $\sigma_{d_{\mathrm{ip}}}$ calculated from the decay track parameter uncertainties. The quality of the description of the data by the simulation is good, including the tails at positive impact-parameter values. The large pulls for secondary $\Lambda$ baryons from cascade decays allow the suppression of this background by means of the kinematic fit.

The uncorrected average rates of reconstructed $V^{0}$ candidates passing the selection cuts per unit pseudorapidity are shown in Fig. 3 as a function of the difference in azimuthal angle $|\Delta \phi|$ between the $V^{0}$ candidate and the leading charged-particle jet. Uncorrected data are compared to PYTHIA events passed through the detailed detector simulation. The dependence of the rates on $|\Delta \phi|$ is qualitatively described by the PYTHIA tunes considered. The simulation underestimates significantly the $V^{0}$ rates in the transverse region. The peak at $|\Delta \phi| \approx 0^{\circ}$ is more pronounced for baryons than for $K_{S}^{0}$ mesons. The simulation indicates that the harder $p_{\mathrm{T}}$ cut applied to the baryon candidates is responsible for this feature; the distributions are similar when the same $p_{\mathrm{T}}$ cut is applied to both $V^{0}$ types.

The backgrounds to the $K_{S}^{0}$ and $\Lambda$ samples are estimated with two methods. The first is based on simulation. Candidates not matched to a generated primary $V^{0}$ of the corresponding type are counted as background. The PYTHIA 6 D6T sample is used. To account for the known deficit of strange particles in the simulation (see Sec. I), the contribution from $K_{S}^{0}$ mesons misidentified as $\Lambda$ baryons is weighted by the ratio of $K_{S}^{0}$ rates measured in nonsingle diffractive events to those in PYTHIA 6 D6T, 1.39 [11]. Similarly, the contribution from misidentified $\Lambda$ baryons 
CMS, pp, $\sqrt{\mathrm{s}}=7 \mathrm{TeV}$
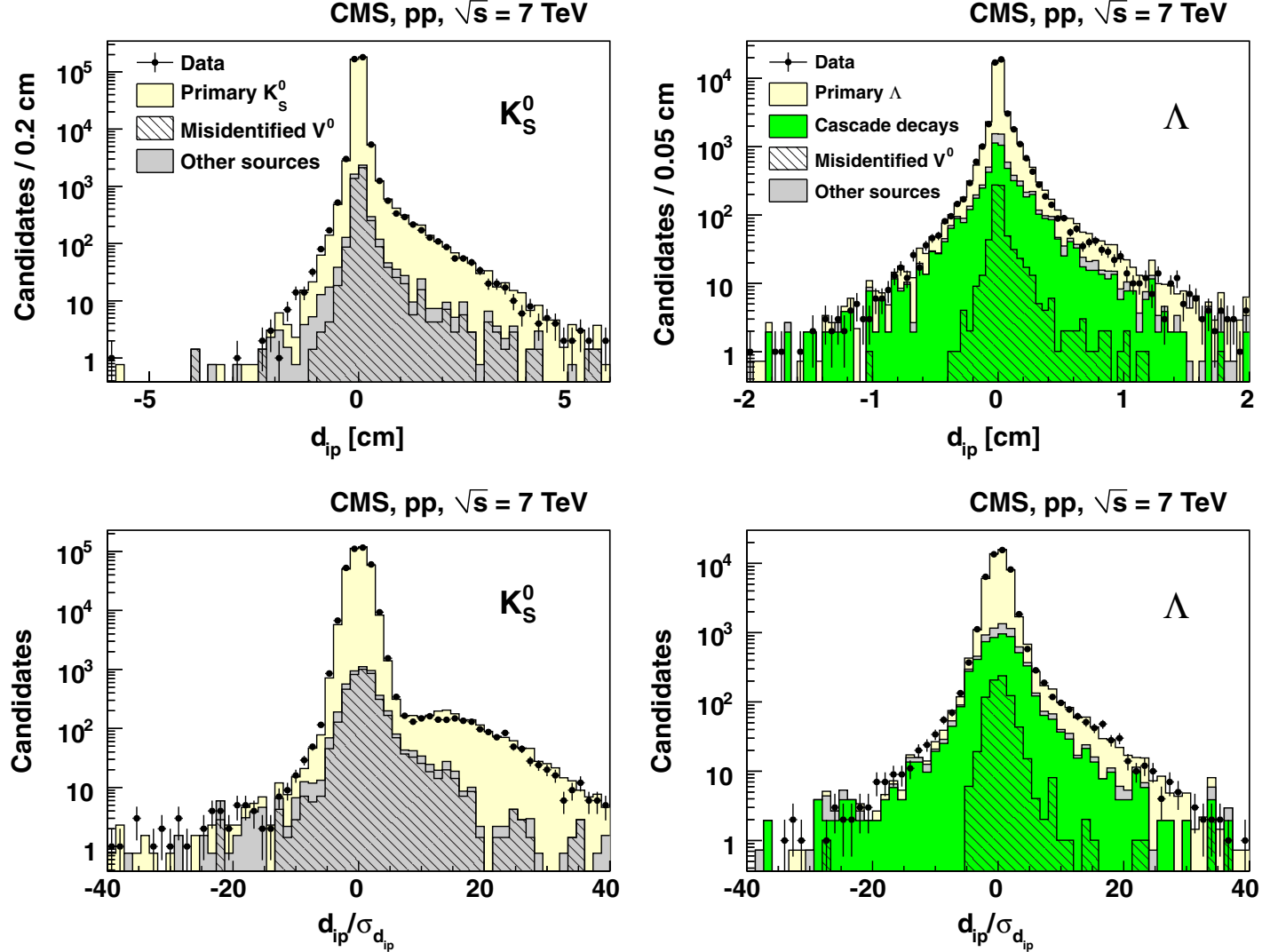

FIG. 2 (color online). Distributions of the signed impact parameter $d_{\text {ip }}$ with respect to the primary vertex, and the corresponding pull distributions, for the most probable particle-type hypothesis determined by the kinematic fit. The black points indicate the data. The histograms show the backgrounds (hatched: misidentified $V^{0}$; green: nonprimary $\Lambda$ from $\Xi$ and $\Omega$ cascade decays; grey: other sources) and the signal (yellow) as predicted by PYTHIA 6 D6T. The PYTHIA prediction is normalized to the data.

is weighted by a factor of 1.85 , and the contribution arising from nonprimary baryons from $\Xi$ and $\Omega$ decays is weighted by the ratio of the measured and simulated $\Xi$ production rates, 2.67 [11].

The second method is based on data. The signal and background contributions are extracted from a fit to the distribution of the kinematic-fit $\chi^{2}$ probability, with signal and background shapes obtained from simulation. Apart from the background normalization, the measured and simulated pull distributions of the constrained variables (Figs. 1 and 2), as well as the measured and simulated $\chi^{2}$-probability distributions (not shown), are in good agreement. These facts, as well as goodness-of-fit tests, validate the approach.

In both methods, the background is estimated as a function of the charged-particle jet $p_{\mathrm{T}}$ for the rate measurements, and as a function of the $V^{0} p_{\mathrm{T}}$ for the $V^{0} p_{\mathrm{T}}$ spectra and the $p_{\mathrm{T}}$ sum measurements. The background estimations from the two methods are in reasonable agreement, and they exhibit the same dependence on the charged-particle jet and $V^{0} p_{\mathrm{T}}$. The final background estimates are computed as the average of the results of the two methods, and the corresponding systematic uncertainties are taken as half the difference of the two results. The background fraction for $K_{S}^{0}$ increases from $(1.5 \pm 1.1) \%$ at charged-particle jet $p_{\mathrm{T}}=1 \mathrm{GeV} / c$ to $(3.3 \pm 1.7) \%$ at charged-particle jet $p_{\mathrm{T}}=10 \mathrm{GeV} / c$ and remains constant at higher charged-particle jet $p_{\mathrm{T}}$. The background is $(8 \pm 2) \%$ for baryons, independent of the charged-particle jet $p_{\mathrm{T}}$.

The $K_{S}^{0}$ and $\Lambda$ raw yields are corrected for purity (defined as 1 -background fraction) as well as for acceptance and reconstruction efficiency. Each $V^{0}$ candidate is weighted by the product of the purity times $\frac{1}{A \times \epsilon}$, where $A$ denotes the acceptance of the cuts on the $V^{0}$ transverse flight distance and on the $p_{\mathrm{T}}, \eta$ of the decay particles, and $\epsilon$ denotes the reconstruction and selection efficiency for accepted $V^{0}$ candidates. The product of acceptance times efficiency is computed in $V^{0}\left(p_{\mathrm{T}}, \eta\right)$ bins from a sample of $50 \times 10^{6}$ PYTHIA 6 D6T minimum-bias events passed through the detailed detector simulation. The average values of the product of acceptance and efficiency in this sample for $K_{S}^{0}$ mesons, and $\Lambda$ and $\bar{\Lambda}$ baryons within the kinematic cuts $\left(|\eta|<2 ; p_{\mathrm{T}}>600 \mathrm{MeV} / c\right.$ for $K_{S}^{0}, p_{\mathrm{T}}>$ $1.5 \mathrm{GeV} / c$ for $\Lambda$ and $\bar{\Lambda}$ ) are $11.3 \%, 8.4 \%$, and $6.6 \%$, respectively, including the branching fractions $\mathcal{B}\left(\mathrm{K}_{S}^{0} \rightarrow\right.$ $\left.\pi^{+} \pi^{-}\right)=69.2 \%$ and $\mathcal{B}\left(\Lambda \rightarrow p \pi^{-}\right)=\mathcal{B}\left(\bar{\Lambda} \rightarrow \bar{p} \pi^{+}\right)=$ $63.9 \%$ [29]. The acceptance depends strongly on the $V^{0}$ 


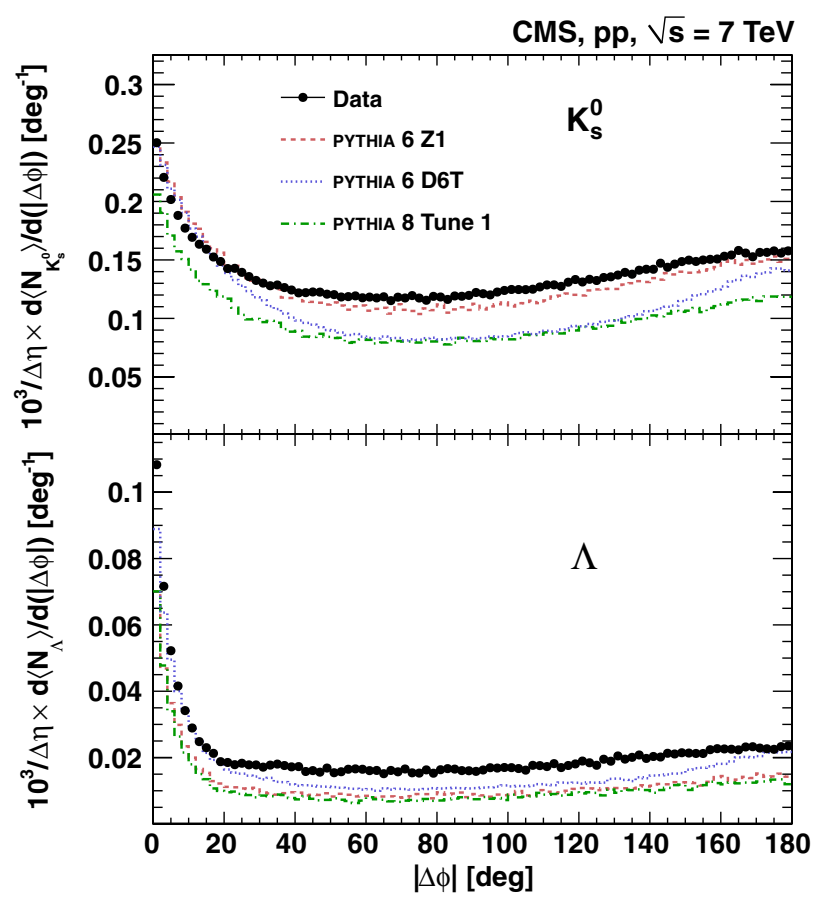

FIG. 3 (color online). Uncorrected average rate of selected $V^{0}$ candidates per event, per degree, and per unit pseudorapidity within $|\eta|<2$, as a function of the difference in azimuthal angle $|\Delta \phi|$ between the $V^{0}$ candidate and the leading chargedparticle jet. Data and detailed simulation of minimum-bias events with different PYTHIA tunes are shown for reconstructed charged-particle jet $p_{\mathrm{T}}>1 \mathrm{GeV} / c$. Top: $K_{S}^{0}$ candidates with $p_{\mathrm{T}}>600 \mathrm{MeV} / c$; bottom: $\Lambda$ candidates with $p_{\mathrm{T}}>$ $1.5 \mathrm{GeV} / c$.

$p_{\mathrm{T}}$, while the efficiency varies by a factor of about 2 in the $V^{0} p_{\mathrm{T}}$ and $\eta$ ranges selected. The smaller efficiency for $\bar{\Lambda}$ baryons than for $\Lambda$ baryons reflects the higher interaction cross section of antiprotons with the detector material compared to that of protons. The corrected $\Lambda$ and $\bar{\Lambda}$ yields are found to be compatible when accounting for the systematic uncertainty due to the modeling of the antiproton cross section in the GEANT4 version used [30] (see Sec. III).

The consistency of the correction method was checked by applying it to all other Monte Carlo samples and comparing the results to the known generated values. Further support to the correction procedure is provided by the fact that the simulation reproduces well several key aspects of the data, most notably the reconstruction efficiency [23,24] and the angular distributions of the $V^{0}$ decay tracks as a function of the $V^{0} p_{\mathrm{T}}$. The reliability of the simulation for $K_{S}^{0}$ and $\Lambda$ reconstruction was checked by comparing the lifetimes obtained from fits to the corrected proper time distributions with the world averages [11]. The stability of the results when varying the $V^{0}$ selection cuts was also checked. The resulting overall contribution of the $V^{0}$ reconstruction to the systematic uncertainty is given in Sec. III.

\section{SYSTEMATIC UNCERTAINTIES}

The main sources of systematic uncertainties are described below, with numerical values summarized in Table I.

Leading charged-particle jet selection: The bias in rates and $p_{\mathrm{T}}$ sums due to mismatches between the reconstructed and the simulated leading charged-particle jets is corrected by means of detailed simulations. The systematic uncertainty is estimated from the residual difference in rates and $p_{\mathrm{T}}$ sums when the reconstructed and the simulated leading charged-particle jets are matched within $\Delta R=0.3$.

Primary vertex selection: The bias caused by the requirement of a minimum track multiplicity at the primary vertex is corrected by means of detailed simulations of minimumbias events with the PYTHIA $6 \mathrm{Z} 1$ tune. The primary charged-particle multiplicity in $7 \mathrm{TeV} p p$ collisions is well described by this tune [1]. The corresponding uncertainty is estimated from the spread of the corrections computed with PYTHIA 6 tunes D6T, Z1 and PYTHIA 8 tune 1.

Modeling of $V^{0}$ reconstruction efficiency: The systematic uncertainty on the $V^{0}$ reconstruction efficiency is estimated from closure tests and from the stability of the results with respect to the $V^{0}$ selection cuts, as described in Sec. II B.

TABLE I. Systematic uncertainties on the measured average $V^{0}$ rates and $p_{\mathrm{T}}$ sums.

\begin{tabular}{|c|c|c|}
\hline \multicolumn{3}{|l|}{ Average rates } \\
\hline Source & $K_{S}^{0}(\%)$ & $\Lambda(\%)$ \\
\hline Leading charged-particle jet selection & 3 & 7 \\
\hline Primary vertex selection & 1 & 1 \\
\hline \multicolumn{3}{|l|}{ Modeling of $V^{0}$ efficiency } \\
\hline Charged-particle jet $p_{\mathrm{T}} \leq 2.5 \mathrm{GeV} / c$ & 3 & 10 \\
\hline Charged-particle jet $p_{\mathrm{T}}>2.5 \mathrm{GeV} / c$ & 3 & 3 \\
\hline Detector material & 3 & 3 \\
\hline GEANT4 cross sections & $\cdots$ & 5 \\
\hline \multicolumn{3}{|l|}{ Statistical uncertainty on $V^{0}$ weights } \\
\hline $600 \mathrm{MeV} / c<p_{\mathrm{T}}^{V^{0}}<700 \mathrm{MeV} / c$ & 0.1 & $\cdots$ \\
\hline $1.5 \mathrm{GeV} / c<p_{\mathrm{T}}^{V^{0}}<1.6 \mathrm{GeV} / c$ & 0.03 & 0.33 \\
\hline $6 \mathrm{GeV} / c<p_{\mathrm{T}}^{V^{0}}<8 \mathrm{GeV} / c$ & 1.4 & 8.3 \\
\hline \multicolumn{3}{|l|}{ Background estimation } \\
\hline Charged-particle jet $p_{\mathrm{T}}=1 \mathrm{GeV} / c$ & 1.1 & 2 \\
\hline Charged-particle jet $p_{\mathrm{T}}=10 \mathrm{GeV} / c$ & 1.7 & 2 \\
\hline \multicolumn{3}{|l|}{ Total } \\
\hline Charged-particle jet $p_{\mathrm{T}}=1 \mathrm{GeV} / c$ & 6 & 14 \\
\hline Charged-particle jet $p_{\mathrm{T}}=10 \mathrm{GeV} / c$ & 6 & 10 \\
\hline \multicolumn{3}{|c|}{ Average $p_{\mathrm{T}}$ sums } \\
\hline Source & $K_{S}^{0}(\%)$ & $\Lambda(\%)$ \\
\hline \multicolumn{3}{|l|}{ Background estimation } \\
\hline$p_{\mathrm{T}}^{V^{0}}=600 \mathrm{MeV} / c$ & 0.1 & . \\
\hline$p_{\mathrm{T}}^{V^{0}}=1.5 \mathrm{GeV} / c$ & 0.8 & 0.3 \\
\hline$p_{\mathrm{T}}^{V^{0}}=8 \mathrm{GeV} / c$ & 3.6 & 4.0 \\
\hline Other sources & as rates & as rates \\
\hline
\end{tabular}


Detector material: The overall mass of the tracker and the relative fractions of the different tracker materials are varied in the simulations, with the requirement that the resulting predicted tracker weight be consistent with the measured weight [31]. The difference between the results thus obtained and the nominal results is taken as a contribution to the systematic uncertainty.

GEANT4 cross sections: A 5\% systematic uncertainty is assigned to the baryon yields, as a result of the known imperfect modeling of the low-energy antiproton interaction cross section in the GEANT4 version used [30].

Statistical uncertainty on the $V^{0}$ yield correction: A small contribution to the total uncertainty stems from the finite size of the sample of minimum-bias events passed through the full detector simulation $\left(50 \times 10^{6}\right.$ events), from which the correction is computed.

Estimation of $V^{0}$ background: The uncertainty on the background remaining after $V^{0}$ identification by means of the kinematic fit is taken as half the difference between the results of the two background estimation methods used.

The uncertainty on the beam spot position and size gives a negligible contribution to the total uncertainty.

\section{RESULTS}

The $V^{0}$ production rates in the transverse region are shown in Fig. 4 as a function of the leading chargedparticle jet $p_{\mathrm{T}}$, and the $V^{0}$ scalar $p_{\mathrm{T}}$ sums in the transverse region are shown in Fig. 5.

The rates and $p_{\mathrm{T}}$ sums exhibit a rise with increasing hard scale, followed by a plateau. The turn-on of the plateau is located at charged-particle jet $p_{\mathrm{T}} \simeq 10 \mathrm{GeV} / c$ for both primary mesons and baryons. Above the turn-on, the rates and $p_{\mathrm{T}}$ sums are essentially constant, implying also a constant strange-particle average $p_{\mathrm{T}}$ above the turn-on.

A comparison can be made with the trends observed for charged primary particles [1] in spite of the different jet reconstruction algorithm used in Ref. [1] (SISCone). The dependence of the UE activity on the charged-particle jet $p_{\mathrm{T}}$ is very similar to that observed for charged primary particles $[1,3,4]$. The most striking feature is that the $p_{\mathrm{T}}$ scale at which the plateau starts, around $10 \mathrm{GeV} / c$ in $p p$ collisions at $\sqrt{s}=7 \mathrm{TeV}$, is independent of the type of primary particle used to probe the UE activity. These observations are consistent with the impact-parameter picture of particle production in hadron collisions [6,7], in which the MPI contribution saturates at scales typical of central collisions.

The PYTHIA $6 \mathrm{Z} 1$ and $\mathrm{Z2}^{*}$ tunes qualitatively reproduce the dependence of the $K_{S}^{0}$ rate and $p_{\mathrm{T}}$ sum on the chargedparticle jet $p_{\mathrm{T}}$, but exhibit a $10 \%-15 \%$ deficit in the yield, independent of the charged-particle jet $p_{\mathrm{T}}$. PYTHIA 8 tune 1 underestimates the activity by about $30 \%$. For the $\Lambda$ baryons, PYTHIA 6 tunes Z1, Z2* and PYTHIA 8 tune 1 underestimate the rates by about $50 \%$. After being tuned to the charged-particle data, PYTHIA 6 Z2* models strangeness
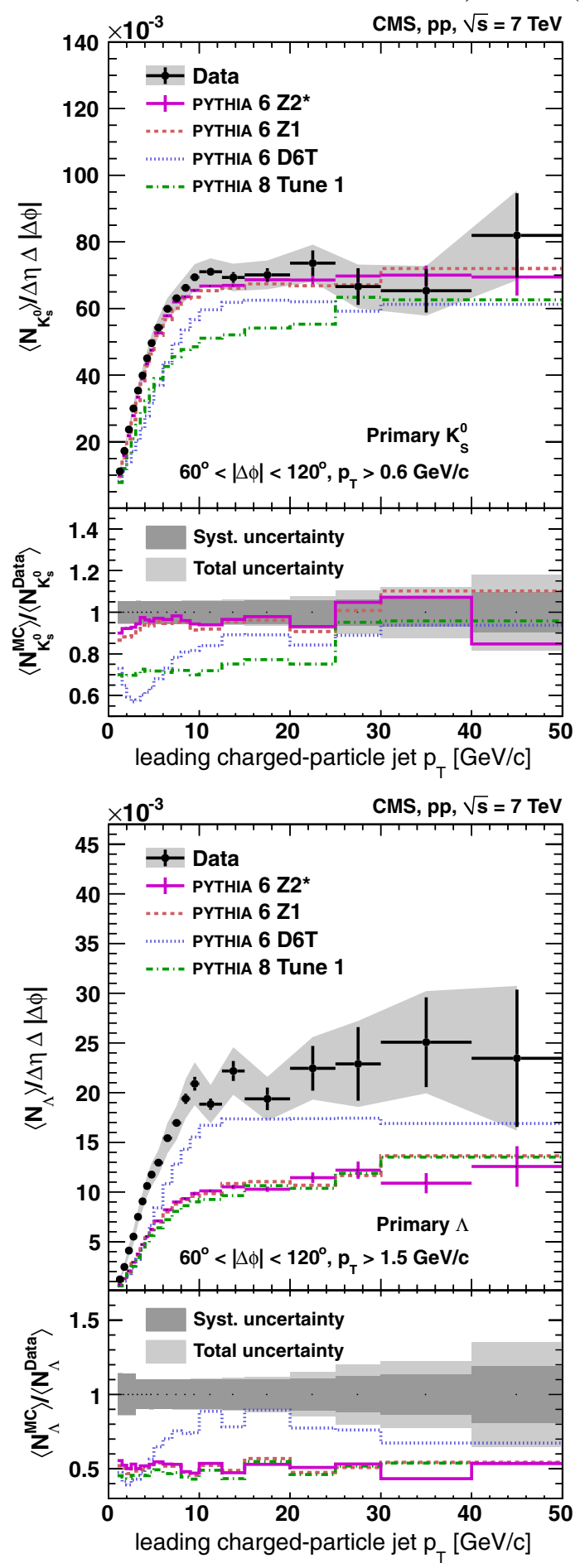

FIG. 4 (color online). Average multiplicity per unit of pseudorapidity and per radian in the transverse region $(|\eta|<2$, $60^{\circ}<|\Delta \phi|<120^{\circ}$ ), as a function of the $p_{\mathrm{T}}$ of the leading charged-particle jet: (top) $K_{S}^{0}$ with $p_{\mathrm{T}}>0.6 \mathrm{GeV} / c$; (bottom) $\Lambda$ with $p_{\mathrm{T}}>1.5 \mathrm{GeV} / c$. Predictions of PYTHIA tunes are compared to the data, and the ratios of simulations to data are shown in the bottom panels. For the data, the statistical uncertainties (error bars) and the quadratic sum of statistical and systematic uncertainties (error band) are shown, while for simulations the uncertainty is only shown for PYTHIA 6 tune $\mathrm{Z2}$, for clarity. 

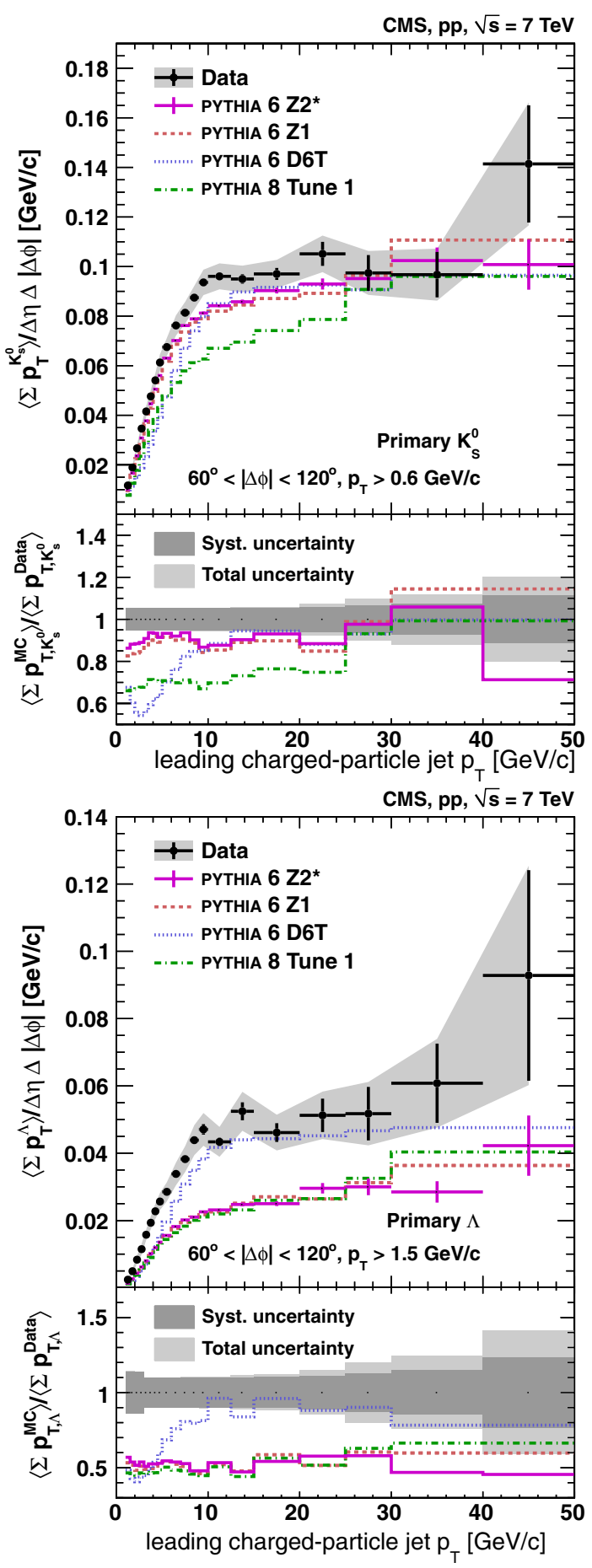

FIG. 5 (color online). Average scalar $p_{\mathrm{T}}$ sum per unit of pseudorapidity and per radian in the transverse region $(|\eta|<2$, $60^{\circ}<|\Delta \phi|<120^{\circ}$ ), as a function of the $p_{\mathrm{T}}$ of the leading charged-particle jet: (top) $K_{S}^{0}$ with $p_{\mathrm{T}}>0.6 \mathrm{GeV} / c$; (bottom) $\Lambda$ with $p_{\mathrm{T}}>1.5 \mathrm{GeV} / c$. Predictions of PYTHIA tunes are compared to the data, and the ratios of simulations to data are shown in the bottom panels. For the data, the statistical uncertainties (error bars) and the quadratic sum of statistical and systematic uncertainties (error band) are shown, while for simulations the uncertainty is only shown for PYTHIA 6 tune $\mathrm{Z} 2 *$, for clarity. production in the UE in a very similar way as Z1, in spite of the different parton distribution set used.

PYTHIA 6 D6T shows a dependence of the activity on the charged-particle jet $p_{\mathrm{T}}$ that differs from that of the data and of the other tunes. In addition, the $V^{0} p_{\mathrm{T}}$ distributions predicted by PYTHIA 6 D6T in the transverse region are in strong disagreement with the data. As an illustration, the $p_{\mathrm{T}}$ spectra are shown in Fig. 6 for events with a
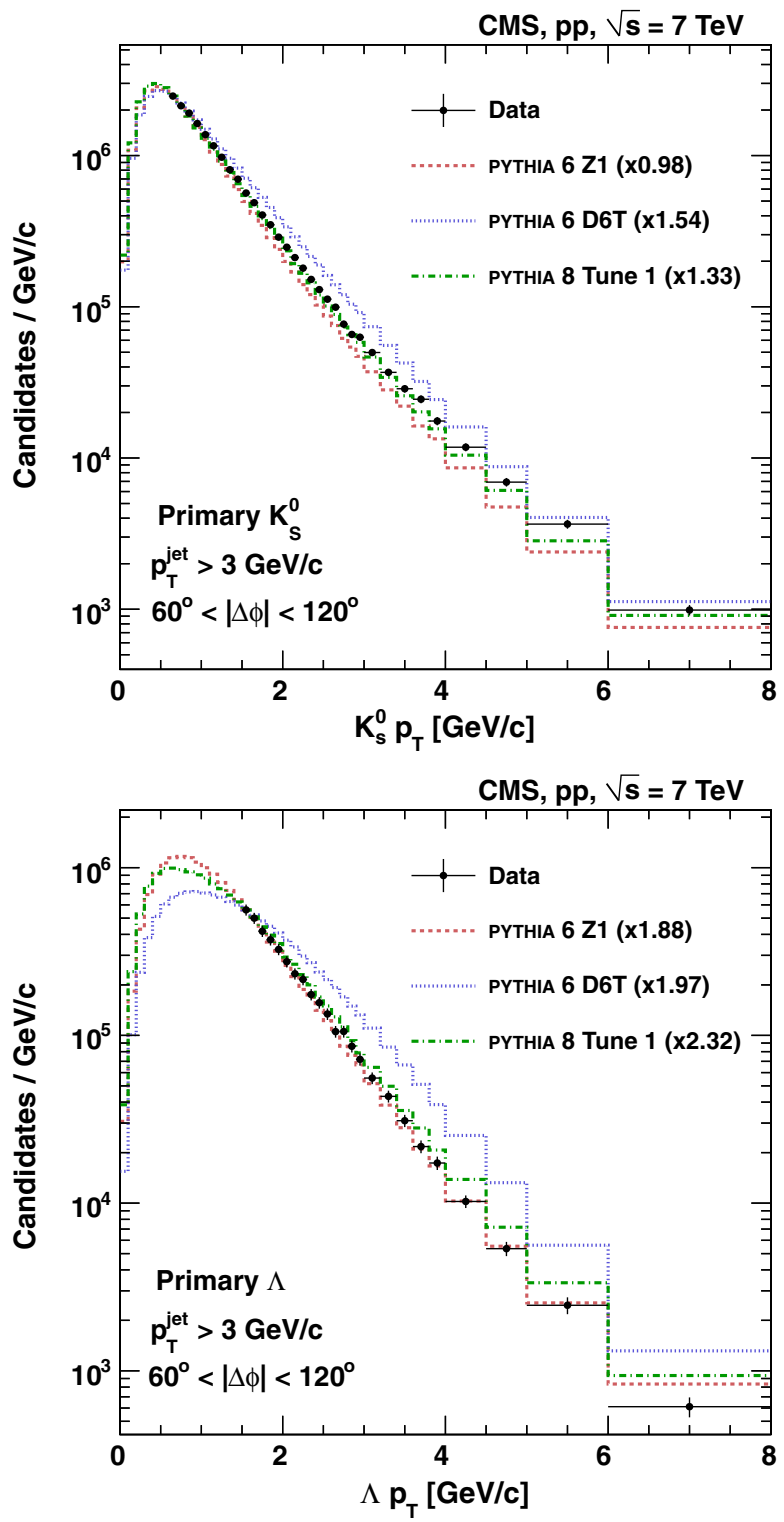

FIG. 6 (color online). $\quad V^{0} p_{\mathrm{T}}$ distributions corrected for selection efficiency and background without a correction to the leading charged-particle jet, in the region transverse to a leading reconstructed charged-particle jet with $p_{\mathrm{T}}>3 \mathrm{GeV} / c$, compared to predictions from different PYTHIA tunes (top: $K_{S}^{0}$; bottom: $\Lambda$ ). Error bars indicate the quadratic sum of the statistical and systematic uncertainties. Simulations are normalized to the first $p_{\mathrm{T}}$ bin in the data, with normalization factors given in parentheses. 

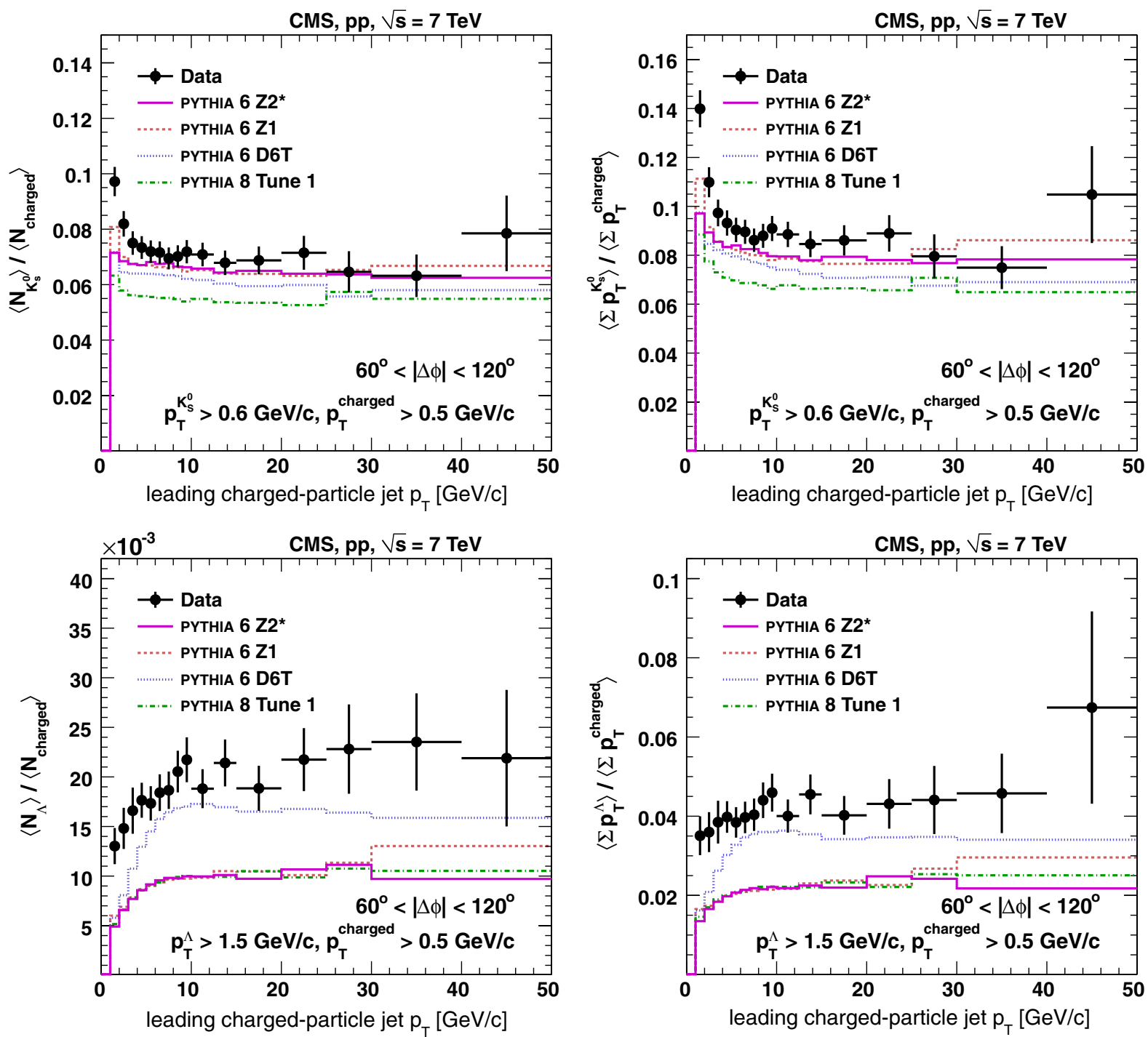

FIG. 7 (color online). Ratios of the average multiplicities and scalar $p_{\mathrm{T}}$ sums for primary $V^{0}$ in the transverse region to the same quantities for primary charged particles [1] as a function of charged-particle jet $p_{\mathrm{T}}$. The error bars indicate the quadratic sum of the statistical and systematic uncertainties.

reconstructed charged-particle jet $p_{\mathrm{T}}>3 \mathrm{GeV} / c$ (without a correction to the leading charged hadron jet). For the $K_{S}^{0}$ case, in the $p_{\mathrm{T}}$ range observed $\left(p_{\mathrm{T}}>600 \mathrm{MeV} / c\right)$, PYTHIA 6 tune D6T shows a much harder spectrum than the data, while tune $\mathrm{Z} 1$ shows a softer spectrum and PYTHIA 8 tune 1 reproduces the shape well. For the $\Lambda$ case, in the $p_{\mathrm{T}}>$ $1.5 \mathrm{GeV} / c$ range, PYTHIA 6 D6T shows a much harder spectrum than the data, while the other simulations describe the data reasonably well.

The ratios of the rates and $p_{\mathrm{T}}$ sums of primary $V^{0}$ mesons to the rates and $p_{\mathrm{T}}$ sums of primary charged particles from Ref. [1] are shown in Fig. 7. The data are integrated over the same pseudorapidity range for strange and charged particles, $|\eta|<2$. The $K_{S}^{0}$ to charged-particle activity ratios are constant in the charged-particle jet $p_{\mathrm{T}}$ range $3-50 \mathrm{GeV} / c$, i.e. almost throughout the whole range studied and, specifically, across the turn-on of the plateau around $10 \mathrm{GeV} / c$. An increase is seen below $3 \mathrm{GeV} / c$. This feature is also present in the simulations but is not as pronounced as in the data, and not in all tunes studied.

The $\Lambda$ to charged-particle activity ratios exhibit a rise for charged-particle jet $p_{\mathrm{T}}<10 \mathrm{GeV} / c$, followed by a plateau. A similar dependence is visible in PYTHIA. Simulations indicate that the rise is related to the observed hardening of the baryon $p_{\mathrm{T}}$ spectrum as the chargedparticle jet $p_{\mathrm{T}}$ increases, combined with the $1.5 \mathrm{GeV} / c$ $p_{\mathrm{T}}$ cut applied to the baryon sample. When the baryon $p_{\mathrm{T}}$ cut is decreased to $0.5 \mathrm{GeV} / c$ as for charged particles, constant ratios are predicted.

Constant strange- to charged-particle activity ratios have thus been measured for $K_{S}^{0}$ mesons for charged-particle jet $p_{\mathrm{T}}>3 \mathrm{GeV} / c$ and for $\Lambda$ baryons for charged-particle jet $p_{\mathrm{T}}>10 \mathrm{GeV} / c$. In addition, as just discussed, when accounting for the acceptance of the baryon $p_{\mathrm{T}}$ cut, a 
constant ratio is also predicted for $\Lambda$ baryons at chargedparticle jet $p_{\mathrm{T}}<10 \mathrm{GeV} / c$. Since the trends observed are very similar for charged and strange particles, as well as for mesons and baryons, the present measurements suggest that hadronization and MPI are decoupled.

\section{CONCLUSIONS}

This paper describes measurements of the underlying event activity in $p p$ collisions at $\sqrt{s}=7 \mathrm{TeV}$, probed through the production of primary $K_{S}^{0}$ mesons and $\Lambda$ baryons. The production of $K_{S}^{0}$ mesons and $\Lambda$ baryons in the kinematic range $p_{\mathrm{T}}^{K_{s}^{0}}>0.6 \mathrm{GeV} / c, p_{\mathrm{T}}^{\Lambda}>1.5 \mathrm{GeV} / c$ and $|\eta|<2$ is analyzed in the transverse region, defined as $60^{\circ}<|\Delta \phi|<120^{\circ}$, with $\Delta \phi$ the difference in azimuthal angle between the leading charged-particle jet and the strange-particle directions. The average multiplicity and the average scalar $p_{\mathrm{T}}$ sum of primary particles per event are studied as a function of the leading chargedparticle jet $p_{\mathrm{T}}$

A steep rise of the underlying event activity is seen with increasing leading jet $p_{\mathrm{T}}$, followed by a "saturation" region for jet $p_{\mathrm{T}}>10 \mathrm{GeV} / c$. This trend and the $p_{\mathrm{T}}$ scale above which saturation occurs are very similar to those observed with charged primary particles. The similarity of the behavior for strange and charged particles is consistent with the impact-parameter picture of multiple parton interactions in $p p$ collisions, in which the centrality of the $p p$ collision and the MPI activity are correlated.

The results are compared to recent tunes of the PYTHIA Monte Carlo event generator. The PYTHIA simulations underestimate the data by $15 \%-30 \%$ for $K_{S}^{0}$ mesons and by about $50 \%$ for $\Lambda$ baryons, a MC deficit similar to that observed for the inclusive strange-particle production in $p p$ collisions.

The constant strange- to charged-particle activity ratios and the similar trends for mesons and baryons indicate that the MPI dynamics is decoupled from parton hadronization, with the latter occurring at a later stage.

\section{ACKNOWLEDGMENTS}

We congratulate our colleagues in the CERN accelerator departments for the excellent performance of the LHC and thank the technical and administrative staffs at CERN and at other CMS institutes for their contributions to the success of the CMS effort. In addition, we gratefully acknowledge the computing centers and personnel of the Worldwide LHC Computing Grid for delivering so effectively the computing infrastructure essential to our analyses. Finally, we acknowledge the enduring support for the construction and operation of the LHC and the CMS detector provided by the following funding agencies: the Austrian Federal Ministry of Science and Research and the Austrian Science Fund; the Belgian Fonds de la Recherche Scientifique, and Fonds voor Wetenschappelijk
Onderzoek; the Brazilian Funding Agencies (CNPq, CAPES, FAPERJ, and FAPESP); the Bulgarian Ministry of Education, Youth and Science; CERN; the Chinese Academy of Sciences, Ministry of Science and Technology, and National Natural Science Foundation of China; the Colombian Funding Agency (COLCIENCIAS); the Croatian Ministry of Science, Education and Sport; the Research Promotion Foundation, Cyprus; the Ministry of Education and Research, Recurrent financing Contract No. SF0690030s09 and European Regional Development Fund, Estonia; the Academy of Finland, Finnish Ministry of Education and Culture, and Helsinki Institute of Physics; the Institut National de Physique Nucléaire et de Physique des Particules/CNRS, and Commissariat à l'Énergie Atomique et aux Énergies Alternatives/CEA, France; the Bundesministerium für Bildung und Forschung, Deutsche Forschungsgemeinschaft, and Helmholtz-Gemeinschaft Deutscher Forschungszentren, Germany; the General Secretariat for Research and Technology, Greece; the National Scientific Research Foundation, and National Office for Research and Technology, Hungary; the Department of Atomic Energy and the Department of Science and Technology, India; the Institute for Studies in Theoretical Physics and Mathematics, Iran; the Science Foundation, Ireland; the Istituto Nazionale di Fisica Nucleare, Italy; the Korean Ministry of Education, Science and Technology and the World Class University program of NRF, Republic of Korea; the Lithuanian Academy of Sciences; the Mexican Funding Agencies (CINVESTAV, CONACYT, SEP, and UASLP-FAI); the Ministry of Science and Innovation, New Zealand; the Pakistan Atomic Energy Commission; the Ministry of Science and Higher Education and the National Science Centre, Poland; the Fundação para a Ciência e a Tecnologia, Portugal; JINR (Armenia, Belarus, Georgia, Ukraine, Uzbekistan); the Ministry of Education and Science of the Russian Federation, the Federal Agency of Atomic Energy of the Russian Federation, Russian Academy of Sciences, and the Russian Foundation for Basic Research; the Ministry of Science and Technological Development of Serbia; the Secretaría de Estado de Investigación, Desarrollo e Innovación and Programa Consolider-Ingenio 2010, Spain; the Swiss Funding Agencies (ETH Board, ETH Zurich, PSI, SNF, UniZH, Canton Zurich, and SER); the National Science Council, Taipei; the Thailand Center of Excellence in Physics, the Institute for the Promotion of Teaching Science and Technology of Thailand and the National Science and Technology Development Agency of Thailand; the Scientific and Technical Research Council of Turkey, and Turkish Atomic Energy Authority; the Science and Technology Facilities Council, UK; and the US Department of Energy and National Science Foundation. Individuals have received support from the Marie-Curie programme and the European Research Council and EPLANET (European Union); the Leventis Foundation; 
the A.P. Sloan Foundation; the Alexander von Humboldt Foundation; the Belgian Federal Science Policy Office; the Fonds pour la Formation à la Recherche dans l'Industrie et dans l'Agriculture (FRIA-Belgium); the Agentschap voor Innovatie door Wetenschap en Technologie (IWT-Belgium); the Ministry of Education, Youth and Sports (MEYS) of
Czech Republic; the Council of Science and Industrial Research, India; the Compagnia di San Paolo (Torino); the HOMING PLUS programme of Foundation for Polish Science, cofinanced by EU, Regional Development Fund; and the Thalis and Aristeia programmes cofinanced by EU-ESF and the Greek NSRF.
[1] CMS Collaboration, J. High Energy Phys. 09 (2011) 109.

[2] R. Field, in Proceedings of the First International Workshop on Multiple Partonic Interactions at the LHC MPI'08, 2008, edited by P. Bartalini and L. Fanó (Perugia, Italy, 2009).

[3] ATLAS Collaboration, Phys. Rev. D 83, 112001 (2011).

[4] CMS Collaboration, Eur. Phys. J. C 70, 555 (2010).

[5] CMS Collaboration, Eur. Phys. J. C 72, 2080 (2012).

[6] T. Sjöstrand and M. van Zijl, Phys. Lett. B 188, 149 (1987).

[7] L. Frankfurt, M. Strikman, and C. Weiss, Phys. Rev. D 83, 054012 (2011).

[8] ALICE Collaboration, Phys. Lett. B 712, 309 (2012).

[9] ALICE Collaboration, arXiv:1211.7298.

[10] ATLAS Collaboration, Phys. Rev. D 85, 012001 (2012).

[11] CMS Collaboration, J. High Energy Phys. 05 (2011) 064.

[12] T. Sjöstrand, S. Mrenna, and P.Z. Skands, J. High Energy Phys. 05 (2006) 026.

[13] T. Sjöstrand, S. Mrenna, and P.Z. Skands, Comput. Phys. Commun. 178, 852 (2008).

[14] R. Field, Acta Phys. Pol. B 39, 2611 (2008).

[15] A. Buckley, H. Hoeth, H. Lacker, H. Schulz, and J. E. von Seggern, Eur. Phys. J. C 65, 331 (2010).

[16] H.-J. Drescher, J. Aichelin, and K. Werner, Phys. Rev. D 65, 057501 (2002).

[17] N. Armesto et al., J. Phys. G 35, 054001 (2008).

[18] CMS Collaboration, JINST 3, S08004 (2008).
[19] W. Waltenberger, R. Frühwirth, and P. Vanlaer, J. Phys. G 34, N343 (2007).

[20] M. Cacciari and G. P. Salam, Phys. Lett. B 641, 57 (2006).

[21] M. Cacciari, G. P. Salam, and G. Soyez, J. High Energy Phys. 04 (2008) 063.

[22] S. Agostinelli et al. (GEANT4), Nucl. Instrum. Methods Phys. Res., Sect. A 506, 250 (2003).

[23] CMS Collaboration, CMS Physics Analysis Summary Report No. CMS-PAS-TRK-10-005, 2010, http:// cdsweb.cern.ch/record/1279383.

[24] CMS Collaboration, CMS Physics Analysis Summary Report No. CMS-PAS-TRK-10-002, 2010, http:// cdsweb.cern.ch/record/1279139.

[25] CMS Collaboration, Eur. Phys. J. C 70, 1165 (2010).

[26] R. Field, arXiv:1010.3558.

[27] R. Corke and T. Sjöstrand, J. High Energy Phys. 03 (2011) 032.

[28] J. Pumplin, D. R. Stump, J. Huston, H.-L. Lai, P. Nadolsky, and W.-K. Tung, J. High Energy Phys. 07 (2002) 012.

[29] J. Beringer et al. (Particle Data Group), Phys. Rev. D 86, 010001 (2012).

[30] ALICE Collaboration, Phys. Rev. Lett. 105, 072002 (2010).

[31] E. Migliore and G. Sguazzoni, CMS NOTE Report No. CMS-NOTE-2010-010, 2010.

S. Chatrchyan, ${ }^{1}$ V. Khachatryan, ${ }^{1}$ A. M. Sirunyan, ${ }^{1}$ A. Tumasyan, ${ }^{1}$ W. Adam,${ }^{2}$ T. Bergauer, ${ }^{2}$ M. Dragicevic, ${ }^{2}$ J. Erö, ${ }^{2}$ C. Fabjan, ${ }^{2, b}$ M. Friedl, ${ }^{2}$ R. Frühwirth, ${ }^{2, b}$ V. M. Ghete, ${ }^{2}$ N. Hörmann, ${ }^{2}$ J. Hrubec, ${ }^{2}$ M. Jeitler, ${ }^{2, b}$ W. Kiesenhofer, ${ }^{2}$ V. Knünz, ${ }^{2}$ M. Krammer, ${ }^{2, \mathrm{~b}}$ I. Krätschmer, ${ }^{2}$ D. Liko, ${ }^{2}$ I. Mikulec, ${ }^{2}$ D. Rabady ${ }^{2, \mathrm{c}}$ B. Rahbaran, ${ }^{2}$ C. Rohringer, ${ }^{2}$ H. Rohringer, ${ }^{2}$ R. Schöfbeck, ${ }^{2}$ J. Strauss, ${ }^{2}$ A. Taurok, ${ }^{2}$ W. Treberer-Treberspurg, ${ }^{2}$ W. Waltenberger, ${ }^{2}$ C.-E. Wulz, ${ }^{2, b}$ V. Mossolov ${ }^{3}$ N. Shumeiko, ${ }^{3}$ J. Suarez Gonzalez, ${ }^{3}$ S. Alderweireldt, ${ }^{4}$ M. Bansal,${ }^{4}$ S. Bansal, ${ }^{4}$ T. Cornelis, ${ }^{4}$ E. A. De Wolf, ${ }^{4}$ X. Janssen, ${ }^{4}$ A. Knutsson, ${ }^{4}$ S. Luyckx ${ }^{4}$ L. Mucibello, ${ }^{4}$ S. Ochesanu ${ }^{4}$ B. Roland, ${ }^{4}$ R. Rougny, ${ }^{4}$ H. Van Haevermaet, ${ }^{4}$ P. Van Mechelen, ${ }^{4}$ N. Van Remortel, ${ }^{4}$ A. Van Spilbeeck, ${ }^{4}$ F. Blekman, ${ }^{5}$ S. Blyweert, ${ }^{5}$ J. D'Hondt, ${ }^{5}$ A. Kalogeropoulos, ${ }^{5}$ J. Keaveney, ${ }^{5}$ M. Maes, ${ }^{5}$ A. Olbrechts, ${ }^{5}$ S. Tavernier, ${ }^{5}$ W. Van Doninck, ${ }^{5}$ P. Van Mulders, ${ }^{5}$ G. P. Van Onsem, ${ }^{5}$ I. Villella,${ }^{5}$ B. Clerbaux,${ }^{6}$ G. De Lentdecker, ${ }^{6}$ L. Favart, ${ }^{6}$ A. P. R. Gay, ${ }^{6}$ T. Hreus, ${ }^{6}$ A. Léonard, ${ }^{6}$ P.E. Marage, ${ }^{6}$ A. Mohammadi, ${ }^{6}$ L. Perniè, ${ }^{6}$ T. Reis, ${ }^{6}$ T. Seva, ${ }^{6}$ L. Thomas, ${ }^{6}{ }^{6}$. Vander Velde, ${ }^{6}$ P. Vanlaer, ${ }^{6}$ J. Wang, ${ }^{6}$ V. Adler, ${ }^{7}$ K. Beernaert, ${ }^{7}$ L. Benucci, ${ }^{7}$ A. Cimmino, ${ }^{7}$ S. Costantini, ${ }^{7}$ S. Dildick, ${ }^{7}$ G. Garcia, ${ }^{7}$ B. Klein, ${ }^{7}$ J. Lellouch, ${ }^{7}$ A. Marinov, ${ }^{7}$ J. Mccartin, ${ }^{7}$ A. A. Ocampo Rios, ${ }^{7}$ D. Ryckbosch, ${ }^{7}$ M. Sigamani, ${ }^{7}$ N. Strobbe, ${ }^{7}$ F. Thyssen, ${ }^{7}$ M. Tytgat, ${ }^{7}$ S. Walsh, ${ }^{7}$ E. Yazgan,${ }^{7}$ N. Zaganidis, ${ }^{7}$ S. Basegmez, ${ }^{8}$ C. Beluffi, ${ }^{8, d}$ G. Bruno, ${ }^{8}$ R. Castello, ${ }^{8}$

A. Caudron, ${ }^{8}$ L. Ceard,${ }^{8}$ C. Delaere,${ }^{8}$ T. du Pree, ${ }^{8}$ D. Favart, ${ }^{8}$ L. Forthomme, ${ }^{8}$ A. Giammanco, ${ }^{8, e}$ J. Hollar, ${ }^{8}$ V. Lemaitre, ${ }^{8}$ J. Liao, ${ }^{8}$ O. Militaru, ${ }^{8}$ C. Nuttens, ${ }^{8}$ D. Pagano, ${ }^{8}$ A. Pin, ${ }^{8}$ K. Piotrzkowski, ${ }^{8}$ A. Popov, ${ }^{8, f}$ M. Selvaggi, ${ }^{8}$ J. M. Vizan Garcia, ${ }^{8}$ N. Beliy, ${ }^{9}$ T. Caebergs, ${ }^{9}$ E. Daubie, ${ }^{9}$ G. H. Hammad, ${ }^{9}$ G. A. Alves, ${ }^{10}$ M. Correa Martins Junior, ${ }^{10}$ T. Martins, ${ }^{10}$ M. E. Pol, ${ }^{10}$ M. H. G. Souza, ${ }^{10}$ W. L. Aldá Júnior, ${ }^{11}$ W. Carvalho, ${ }^{11}$ J. Chinellato, ${ }^{11, g}$ A. Custódio, ${ }^{11}$ 
E. M. Da Costa, ${ }^{11}$ D. De Jesus Damiao,${ }^{11}$ C. De Oliveira Martins, ${ }^{11}$ S. Fonseca De Souza,${ }^{11}$ H. Malbouisson, ${ }^{11}$ M. Malek, ${ }^{11}$ D. Matos Figueiredo, ${ }^{11}$ L. Mundim,${ }^{11}$ H. Nogima, ${ }^{11}$ W. L. Prado Da Silva, ${ }^{11}$ A. Santoro, ${ }^{11}$ L. Soares Jorge, ${ }^{11}$ A. Sznajder, ${ }^{11}$ E. J. Tonelli Manganote, ${ }^{11, \mathrm{~g}}$ A. Vilela Pereira, ${ }^{11}$ T. S. Anjos ${ }^{12 \mathrm{~b}}$ C. A. Bernardes, ${ }^{12 \mathrm{~b}}$ F. A. Dias, ${ }^{12 a, h}$ T. R. Fernandez Perez Tomei, ${ }^{12 a}$ E. M. Gregores, ${ }^{12 b}$ C. Lagana, ${ }^{12 a}$ F. Marinho, ${ }^{12 a}$ P. G. Mercadante, ${ }^{12 b}$ S. F. Novaes, ${ }^{12 a}$ Sandra S. Padula, ${ }^{12 a}$ V. Genchev,${ }^{13, c}$ P. Iaydjiev, ${ }^{13, c}$ S. Piperov,${ }^{13}$ M. Rodozov,${ }^{13}$ G. Sultanov, ${ }^{13}$ M. Vutova, ${ }^{13}$ A. Dimitrov ${ }^{14}$ R. Hadjiiska, ${ }^{14}$ V. Kozhuharov,${ }^{14}$ L. Litov, ${ }^{14}$ B. Pavlov, ${ }^{14}$ P. Petkov, ${ }^{14}$ J. G. Bian, ${ }^{15}$ G. M. Chen, ${ }^{15}$ H. S. Chen, ${ }^{15}$ C. H. Jiang, ${ }^{15}$ D. Liang, ${ }^{15}$ S. Liang, ${ }^{15}$ X. Meng, ${ }^{15}$ J. Tao, ${ }^{15}$ J. Wang, ${ }^{15}$ X. Wang, ${ }^{15}$ Z. Wang, ${ }^{15} \mathrm{H}$. Xiao, ${ }^{15} \mathrm{M}$. Xu, ${ }^{15} \mathrm{C}$. Asawatangtrakuldee, ${ }^{16}$ Y. Ban, ${ }^{16}$ Y. Guo $,{ }^{16} \mathrm{Q} . \mathrm{Li},{ }^{16} \mathrm{~W} . \mathrm{Li},{ }^{16} \mathrm{~S} . \mathrm{Liu},{ }^{16} \mathrm{Y}$. Mao, ${ }^{16}$

S. J. Qian, ${ }^{16}$ D. Wang, ${ }^{16}$ L. Zhang, ${ }^{16}$ W. Zou, ${ }^{16}$ C. Avila, ${ }^{17}$ C. A. Carrillo Montoya, ${ }^{17}$ J. P. Gomez, ${ }^{17}$

B. Gomez Moreno, ${ }^{17}$ J.C. Sanabria,${ }^{17}$ N. Godinovic, ${ }^{18}$ D. Lelas, ${ }^{18}$ R. Plestina, ${ }^{18, i}$ D. Polic,${ }^{18}$ I. Puljak, ${ }^{18}$ Z. Antunovic, ${ }^{19}$ M. Kovac, ${ }^{19}$ V. Brigljevic, ${ }^{20}$ S. Duric, ${ }^{20}$ K. Kadija,${ }^{20}$ J. Luetic, ${ }^{20}$ D. Mekterovic, ${ }^{20}$ S. Morovic, ${ }^{20}$ L. Tikvica, ${ }^{20}$ A. Attikis, ${ }^{21}$ G. Mavromanolakis,${ }^{21}$ J. Mousa,${ }^{21}$ C. Nicolaou,${ }^{21}$ F. Ptochos, ${ }^{21}$ P. A. Razis,${ }^{21}$ M. Finger,${ }^{22}$ M. Finger, Jr., ${ }^{22}$ Y. Assran, ${ }^{23, j}$ A. Ellithi Kamel,${ }^{23, k}$ M. A. Mahmoud, ${ }^{23,1}$ A. Mahrous, ${ }^{23, m}$ A. Radi, ${ }^{23, n, o}$ M. Kadastik, ${ }^{24}$ M. Müntel,${ }^{24}$ M. Murumaa,${ }^{24}$ M. Raidal,${ }^{24}$ L. Rebane,${ }^{24}$ A. Tiko, ${ }^{24}$ P. Eerola ${ }^{25}$ G. Fedi, ${ }^{25}$ M. Voutilainen, ${ }^{25}$ J. Härkönen, ${ }^{26}$ V. Karimäki, ${ }^{26}$ R. Kinnunen, ${ }^{26}$ M. J. Kortelainen, ${ }^{26}$ T. Lampén, ${ }^{26}$ K. Lassila-Perini, ${ }^{26}$ S. Lehti, ${ }^{26}$

T. Lindén, ${ }^{26}$ P. Luukka, ${ }^{26}$ T. Mäenpää, ${ }^{26}$ T. Peltola,${ }^{26}$ E. Tuominen, ${ }^{26}$ J. Tuominiemi,${ }^{26}$ E. Tuovinen, ${ }^{26}$

L. Wendland, ${ }^{26}$ A. Korpela, ${ }^{27}$ T. Tuuva, ${ }^{27}$ M. Besancon, ${ }^{28}$ S. Choudhury, ${ }^{28}$ F. Couderc, ${ }^{28}$ M. Dejardin,,${ }^{28}$ D. Denegri, ${ }^{28}$ B. Fabbro, ${ }^{28}$ J. L. Faure, ${ }^{28}$ F. Ferri, ${ }^{28}$ S. Ganjour, ${ }^{28}$ A. Givernaud,${ }^{28}$ P. Gras, ${ }^{28}$

G. Hamel de Monchenault, ${ }^{28}$ P. Jarry, ${ }^{28}$ E. Locci, ${ }^{28}$ J. Malcles, ${ }^{28}$ L. Millischer ${ }^{28}$ A. Nayak, ${ }^{28}$ J. Rander,${ }^{28}$ A. Rosowsky, ${ }^{28}$ M. Titov, ${ }^{28}$ S. Baffioni, ${ }^{29}$ F. Beaudette, ${ }^{29}$ L. Benhabib,${ }^{29}$ L. Bianchini, ${ }^{29}$ M. Bluj, ${ }^{29, p}$ P. Busson, ${ }^{29}$ C. Charlot,${ }^{29}$ N. Daci, ${ }^{29}$ T. Dahms, ${ }^{29}$ M. Dalchenko, ${ }^{29}$ L. Dobrzynski, ${ }^{29}$ A. Florent, ${ }^{29}$ R. Granier de Cassagnac, ${ }^{29}$ M. Haguenauer, ${ }^{29}$ P. Miné, ${ }^{29}$ C. Mironov, ${ }^{29}$ I. N. Naranjo, ${ }^{29}$ M. Nguyen, ${ }^{29}$ C. Ochando, ${ }^{29}$ P. Paganini, ${ }^{29}$ D. Sabes, ${ }^{29}$ R. Salerno, ${ }^{29}$ Y. Sirois,${ }^{29}$ C. Veelken, ${ }^{29}$ A. Zabi, ${ }^{29}$ J.-L. Agram, ${ }^{30, q}$ J. Andrea,${ }^{30}$ D. Bloch, ${ }^{30}$ D. Bodin,${ }^{30}$ J.-M. Brom, ${ }^{30}$ E. C. Chabert, ${ }^{30}$ C. Collard, ${ }^{30}$ E. Conte, ${ }^{30, q}$ F. Drouhin, ${ }^{30, q}$ J.-C. Fontaine, ${ }^{30, q}$ D. Gelé,,${ }^{30}$ U. Goerlach, ${ }^{30}$ C. Goetzmann, ${ }^{30}$ P. Juillot, ${ }^{30}$ A.-C. Le Bihan, ${ }^{30}$ P. Van Hove,${ }^{30}$ S. Gadrat,${ }^{31}$ S. Beauceron, ${ }^{32}$ N. Beaupere, ${ }^{32}$ G. Boudoul, ${ }^{32}$ S. Brochet, ${ }^{32}$ J. Chasserat, ${ }^{32}$ R. Chierici, ${ }^{32}$ D. Contardo ${ }^{32}$ P. Depasse, ${ }^{32}$ H. El Mamouni, ${ }^{32}$ J. Fay, ${ }^{32}$ S. Gascon, ${ }^{32}$ M. Gouzevitch, ${ }^{32}$ B. Ille, ${ }^{32}$ T. Kurca,${ }^{32}$ M. Lethuillier, ${ }^{32}$ L. Mirabito, ${ }^{32}$ S. Perries, ${ }^{32}$ L. Sgandurra, ${ }^{32}$ V. Sordini, ${ }^{32}$ Y. Tschudi, ${ }^{32}$ M. Vander Donckt, ${ }^{32}$ P. Verdier, ${ }^{32}$ S. Viret, ${ }^{32}$ Z. Tsamalaidze, ${ }^{33, r}$ C. Autermann, ${ }^{34}$ S. Beranek, ${ }^{34}$ B. Calpas, ${ }^{34}$ M. Edelhoff,${ }^{34}$ L. Feld,${ }^{34}$ N. Heracleous, ${ }^{34}$ O. Hindrichs,${ }^{34}$ K. Klein, ${ }^{34}$ J. Merz, ${ }^{34}$ A. Ostapchuk, ${ }^{34}$ A. Perieanu, ${ }^{34}$ F. Raupach, ${ }^{34}$ J. Sammet, ${ }^{34}$ S. Schael, ${ }^{34}$ D. Sprenger, ${ }^{34}$ H. Weber,${ }^{34}$ B. Wittmer, ${ }^{34}$ V. Zhukov, ${ }^{34, f}$ M. Ata, ${ }^{35}$ J. Caudron, ${ }^{35}$ E. Dietz-Laursonn, ${ }^{35}$ D. Duchardt, ${ }^{35}$ M. Erdmann,,${ }^{35}$ R. Fischer, ${ }^{35}$ A. Güth ${ }^{35}$ T. Hebbeker, ${ }^{35}$ C. Heidemann, ${ }^{35}$ K. Hoepfner, ${ }^{35}$ D. Klingebiel, ${ }^{35}$ P. Kreuzer, ${ }^{35}$ M. Merschmeyer, ${ }^{35}$ A. Meyer, ${ }^{35}$ M. Olschewski, ${ }^{35}$ K. Padeken, ${ }^{35}$ P. Papacz, ${ }^{35}$ H. Pieta, ${ }^{35}$ H. Reithler, ${ }^{35}$ S. A. Schmitz, ${ }^{35}$ L. Sonnenschein,,${ }^{35}$ J. Steggemann, ${ }^{35}$ D. Teyssier, ${ }^{35}$ S. Thüer, ${ }^{35}$ M. Weber, ${ }^{35}$ V. Cherepanov, ${ }^{36}$ Y. Erdogan, ${ }^{36}$ G. Flügge,${ }^{36}$ H. Geenen, ${ }^{36}$ M. Geisler, ${ }^{36}$ W. Haj Ahmad, ${ }^{36}$ F. Hoehle, ${ }^{36}$ B. Kargoll, ${ }^{36}$ T. Kress, ${ }^{36}$ Y. Kuessel, ${ }^{36}$ J. Lingemann, ${ }^{36, c}$ A. Nowack, ${ }^{36}$ I. M. Nugent, ${ }^{36}$ L. Perchalla, ${ }^{36}$ O. Pooth, ${ }^{36}$ A. Stahl, ${ }^{36}$ M. Aldaya Martin, ${ }^{37}$ I. Asin, ${ }^{37}$ N. Bartosik,,${ }^{37}$ J. Behr, ${ }^{37}$ W. Behrenhoff, ${ }^{37}$ U. Behrens, ${ }^{37}$ M. Bergholz, ${ }^{37, s}$ A. Bethani, ${ }^{37}$ K. Borras,${ }^{37}$ A. Burgmeier, ${ }^{37}$ A. Cakir, ${ }^{37}$

L. Calligaris, ${ }^{37}$ A. Campbell, ${ }^{37}$ F. Costanza, ${ }^{37}$ C. Diez Pardos,${ }^{37}$ T. Dorland, ${ }^{37}$ G. Eckerlin, ${ }^{37}$ D. Eckstein, ${ }^{37}$ G. Flucke,${ }^{37}$ A. Geiser, ${ }^{37}$ I. Glushkov, ${ }^{37}$ P. Gunnellini, ${ }^{37}$ S. Habib,${ }^{37}$ J. Hauk,${ }^{37}$ G. Hellwig,,${ }^{37}$ H. Jung, ${ }^{37}$ M. Kasemann, ${ }^{37}$ P. Katsas, ${ }^{37}$ C. Kleinwort, ${ }^{37}$ H. Kluge, ${ }^{37}$ M. Krämer, ${ }^{37}$ D. Krücker, ${ }^{37}$ E. Kuznetsova, ${ }^{37}$ W. Lange, ${ }^{37}$ J. Leonard, ${ }^{37}$ K. Lipka,${ }^{37}$ W. Lohmann, ${ }^{37, s}$ B. Lutz,${ }^{37}$ R. Mankel,${ }^{37}$ I. Marfin, ${ }^{37}$ I.-A. Melzer-Pellmann, ${ }^{37}$ A. B. Meyer,${ }^{37}$ J. Mnich,${ }^{37}$ A. Mussgiller, ${ }^{37}$ S. Naumann-Emme, ${ }^{37}$ O. Novgorodova, ${ }^{37}$ F. Nowak,${ }^{37}$ J. Olzem, ${ }^{37}$ H. Perrey, ${ }^{37}$ A. Petrukhin, ${ }^{37}$ D. Pitzl,${ }^{37}$ R. Placakyte, ${ }^{37}$ A. Raspereza,${ }^{37}$ P. M. Ribeiro Cipriano, ${ }^{37}$ C. Riedl,,${ }^{37}$ E. Ron, ${ }^{37}$ M. Ö. Sahin, ${ }^{37}$ J. Salfeld-Nebgen, ${ }^{37}$ R. Schmidt, ${ }^{37, s}$ T. Schoerner-Sadenius, ${ }^{37}$ N. Sen, ${ }^{37}$ M. Stein, ${ }^{37}$ R. Walsh, ${ }^{37}$ C. Wissing, ${ }^{37}$ V. Blobel,${ }^{38}$ H. Enderle, ${ }^{38}$ J. Erfle, ${ }^{38}$ U. Gebbert, ${ }^{38}$ M. Görner, ${ }^{38}$ M. Gosselink, ${ }^{38}$ J. Haller, ${ }^{38}$ K. Heine, ${ }^{38}$ R. S. Höing, ${ }^{38}$ G. Kaussen, ${ }^{38}$ H. Kirschenmann, ${ }^{38}$ R. Klanner, ${ }^{38}$ R. Kogler, ${ }^{38}$ J. Lange, ${ }^{38}$ I. Marchesini, ${ }^{38}$ T. Peiffer, ${ }^{38}$ N. Pietsch, ${ }^{38}$ D. Rathjens, ${ }^{38}$ C. Sander,${ }^{38}$ H. Schettler, ${ }^{38}$ P. Schleper, ${ }^{38}$ E. Schlieckau, ${ }^{38}$ A. Schmidt, ${ }^{38}$ M. Schröder ${ }^{38}$ T. Schum, ${ }^{38}$ M. Seidel, ${ }^{38}$ J. Sibille, ${ }^{38, t}$ V. Sola ${ }^{38}$ H. Stadie, ${ }^{38}$ G. Steinbrück, ${ }^{38}$ J. Thomsen, ${ }^{38}$ D. Troendle, ${ }^{38}$ L. Vanelderen, ${ }^{38}$ C. Barth,${ }^{39}$ C. Baus, ${ }^{39}$ J. Berger, ${ }^{39}$ C. Böser, ${ }^{39}$ T. Chwalek,${ }^{39}$ W. De Boer, ${ }^{39}$ A. Descroix, ${ }^{39}$ A. Dierlamm, ${ }^{39}$ M. Feindt,${ }^{39}$ M. Guthoff,,${ }^{39, c}$ C. Hackstein, ${ }^{39}$ F. Hartmann, ${ }^{39, c}$ T. Hauth,${ }^{39, \mathrm{c}}$ M. Heinrich, ${ }^{39}$ H. Held,${ }^{39}$ K. H. Hoffmann, ${ }^{39}$ U. Husemann, ${ }^{39}$ I. Katkov, ${ }^{39, f}$ J. R. Komaragiri, ${ }^{39}$ 
A. Kornmayer ${ }^{39, c}$ P. Lobelle Pardo, ${ }^{39}$ D. Martschei, ${ }^{39}$ S. Mueller, ${ }^{39}$ Th. Müller, ${ }^{39}$ M. Niegel,,${ }^{39}$ A. Nürnberg, ${ }^{39}$ O. Oberst, ${ }^{39}$ J. Ott, ${ }^{39}$ G. Quast,${ }^{39}$ K. Rabbertz,${ }^{39}$ F. Ratnikov, ${ }^{39}$ S. Röcker, ${ }^{39}$ F.-P. Schilling, ${ }^{39}$ G. Schott, ${ }^{39}$ H. J. Simonis, ${ }^{39}$ F. M. Stober, ${ }^{39}$ R. Ulrich,${ }^{39}$ J. Wagner-Kuhr, ${ }^{39}$ S. Wayand ${ }^{39}$ T. Weiler, ${ }^{39}$ M. Zeise, ${ }^{39}$ G. Anagnostou, ${ }^{40}$ G. Daskalakis, ${ }^{40}$ T. Geralis,${ }^{40}$ S. Kesisoglou, ${ }^{40}$ A. Kyriakis, ${ }^{40}$ D. Loukas, ${ }^{40}$ A. Markou, ${ }^{40}$ C. Markou, ${ }^{40}$ E. Ntomari, ${ }^{40}$ L. Gouskos,${ }^{41}$ T. J. Mertzimekis, ${ }^{41}$ A. Panagiotou, ${ }^{41}$ N. Saoulidou, ${ }^{41}$ E. Stiliaris,${ }^{41}$ X. Aslanoglou, ${ }^{42}$ I. Evangelou, ${ }^{42}$ G. Flouris,${ }^{42}$ C. Foudas, ${ }^{42}$ P. Kokkas, ${ }^{42}$ N. Manthos, ${ }^{42}$ I. Papadopoulos, ${ }^{42}$ E. Paradas, ${ }^{42}$ G. Bencze, ${ }^{43}$ C. Hajdu, ${ }^{43}$ P. Hidas, ${ }^{43}$ D. Horvath, ${ }^{43, u}$ B. Radics, ${ }^{43}$ F. Sikler, ${ }^{43}$ V. Veszpremi, ${ }^{43}$ G. Vesztergombi, ${ }^{43, v}$ A. J. Zsigmond ${ }^{43}$ N. Beni, ${ }^{44}$ S. Czellar, ${ }^{44}$ J. Molnar, ${ }^{44}$ J. Palinkas, ${ }^{44}$ Z. Szillasi ${ }^{44}$ J. Karancsi, ${ }^{45}$ P. Raics ${ }^{45}$ Z. L. Trocsanyi, ${ }^{45}$ B. Ujvari, ${ }^{45}$ S. B. Beri, ${ }^{46}$ V. Bhatnagar, ${ }^{46}$ N. Dhingra, ${ }^{46}$ R. Gupta, ${ }^{46}$ M. Kaur, ${ }^{46}$ M.Z. Mehta, ${ }^{46}$ M. Mittal, ${ }^{46}$ N. Nishu, ${ }^{46}$ L. K. Saini ${ }^{46}$ A. Sharma ${ }^{46}$ J. B. Singh, ${ }^{46}$ Ashok Kumar, ${ }^{47}$ Arun Kumar, ${ }^{47}$ S. Ahuja, ${ }^{47}$ A. Bhardwaj ${ }^{47}$ B. C. Choudhary, ${ }^{47}$ S. Malhotra,${ }^{47}$ M. Naimuddin, ${ }^{47}$ K. Ranjan,${ }^{47}$ P. Saxena,${ }^{47}$ V. Sharma,${ }^{47}$ R. K. Shivpuri, ${ }^{47}$ S. Banerjee, ${ }^{48}$ S. Bhattacharya,${ }^{48}$ K. Chatterjee,${ }^{48}$ S. Dutta ${ }^{48}$ B. Gomber ${ }^{48}$ Sa. Jain ${ }^{48}$ Sh. Jain, ${ }^{48}$ R. Khurana, ${ }^{48}$ A. Modak, ${ }^{48}$ S. Mukherjee, ${ }^{48}$ D. Roy, ${ }^{48}$ S. Sarkar, ${ }^{48}$ M. Sharan, ${ }^{48}$ A. Abdulsalam, ${ }^{49}$ D. Dutta, ${ }^{49}$ S. Kailas, ${ }^{49}$ V. Kumar, ${ }^{49}$ A. K. Mohanty,${ }^{49, c}$ L. M. Pant, ${ }^{49}$ P. Shukla, ${ }^{49}$ A. Topkar, ${ }^{49}$ T. Aziz,${ }^{50}$ R. M. Chatterjee, ${ }^{50}$ S. Ganguly, ${ }^{50}$ S. Ghosh, ${ }^{50}$ M. Guchait,${ }^{50, w}$ A. Gurtu,${ }^{50, x}$ G. Kole, ${ }^{50}$ S. Kumar,${ }^{50}$ M. Maity, ${ }^{50, y}$ G. Majumder, ${ }^{50}$ K. Mazumdar, ${ }^{50}$ G. B. Mohanty, ${ }^{50}$ B. Parida, ${ }^{50}$ K. Sudhakar,${ }^{50}$ N. Wickramage,${ }^{50, z}$ S. Banerjee, ${ }^{51}$ S. Dugad, ${ }^{51}$ H. Arfaei, ${ }^{52 \text {,aa }}$ H. Bakhshiansohi ${ }^{52}$ S. M. Etesami, ${ }^{52, \text { bb }}$ A. Fahim, ${ }^{52, \text { aa }}$ H. Hesari, ${ }^{52}$ A. Jafari, ${ }^{52}$ M. Khakzad, ${ }^{52}$ M. Mohammadi Najafabadi, ${ }^{52}$ S. Paktinat Mehdiabadi, ${ }^{52}$ B. Safarzadeh, ${ }^{52, c c}$ M. Zeinali, ${ }^{52}$ M. Grunewald ${ }^{53}$ M. Abbrescia ${ }^{54 a, 54 b}$ L. Barbone, ${ }^{54 a, 54 b}$ C. Calabria, ${ }^{54 a, 54 b}$ S. S. Chhibra, ${ }^{54 a, 54 b}$ A. Colaleo, ${ }^{54 a}$

D. Creanza,${ }^{54 a, 54 c}$ N. De Filippis, ${ }^{54 a, 54 c, c}$ M. De Palma,${ }^{54 a, 54 b}$ L. Fiore, ${ }^{54 a}$ G. Iaselli, ${ }^{54 a, 54 c}$ G. Maggi, ${ }^{54 a, 54 c}$ M. Maggi, ${ }^{54 \mathrm{a}}$ B. Marangelli, ${ }^{54 \mathrm{a}, 54 \mathrm{~b}}$ S. My, ${ }^{54 \mathrm{a}, 54 \mathrm{c}}$ S. Nuzzo, ${ }^{54 \mathrm{a}, 54 \mathrm{~b}}$ N. Pacifico, ${ }^{54 \mathrm{a}}$ A. Pompili, ${ }^{54 a, 54 \mathrm{~b}}$ G. Pugliese, ${ }^{54 a, 54 \mathrm{c}}$ G. Selvaggi, ${ }^{54,54 b}$ L. Silvestris, ${ }^{54 a}$ G. Singh, ${ }^{54 a, 54 b}$ R. Venditti, ${ }^{54 a, 54 b}$ P. Verwilligen, ${ }^{54 a}$ G. Zito, ${ }^{54 a}$ G. Abbiendi, ${ }^{55 a}$ A. C. Benvenuti, ${ }^{55 a}$ D. Bonacorsi, ${ }^{55 a, 55 b}$ S. Braibant-Giacomelli, ${ }^{55 a, 55 b}$ L. Brigliadori, ${ }^{55 a, 55 b}$ R. Campanini, ${ }^{55 a, 55 b}$

P. Capiluppi, ${ }^{55 a, 55 b}$ A. Castro, ${ }^{55 a, 55 b}$ F. R. Cavallo, ${ }^{55 a}$ M. Cuffiani, ${ }^{55 a, 55 b}$ G. M. Dallavalle, ${ }^{55 a}$ F. Fabbri, ${ }^{55 a}$ A. Fanfani, ${ }^{55 a, 55 b}$ D. Fasanella, ${ }^{55 a, 55 b}$ P. Giacomelli, ${ }^{55 a}$ C. Grandi, ${ }^{55 a}$ L. Guiducci, ${ }^{55 a, 55 b}$ S. Marcellini, ${ }^{55 a}$ G. Masetti, ${ }^{55 a, c}$ M. Meneghelli, ${ }^{55 a, 55 b}$ A. Montanari, ${ }^{55 a}$ F. L. Navarria, ${ }^{55 a, 55 b}$ F. Odorici, ${ }^{55 a}$ A. Perrotta, ${ }^{55 a}$ F. Primavera, ${ }^{55 a, 55 b}$ A. M. Rossi, ${ }^{55 a, 55 b}$ T. Rovelli, ${ }^{55 a, 55 b}$ G. P. Siroli, ${ }^{55 a, 55 b}$ N. Tosi, ${ }^{55 a, 55 b}$ R. Travaglini, ${ }^{55 a, 55 b}$ S. Albergo, ${ }^{56 a, 56 b}$ M. Chiorboli, ${ }^{56 a, 56 b}$ S. Costa,${ }^{56 a, 56 b}$ F. Giordano, ${ }^{56 a, c}$ R. Potenza, ${ }^{56 a, 56 b}$ A. Tricomi,${ }^{56 a, 56 b}$ C. Tuve, ${ }^{56 a, 56 b}$ G. Barbagli, ${ }^{57 a}$ V. Ciulli, ${ }^{57 a, 57 b}$ C. Civinini, ${ }^{57 a}$ R. D’Alessandro, ${ }^{57 a, 57 b}$ E. Focardi, ${ }^{57 a, 57 b}$ S. Frosali, ${ }^{57,57 b}$ E. Gallo, ${ }^{57 a}$ S. Gonzi, ${ }^{57 a, 57 b}$ V. Gori, ${ }^{57 a, 57 b}$ P. Lenzi, ${ }^{57 a, 57 b}$ M. Meschini, ${ }^{57 a}$ S. Paoletti, ${ }^{57 a}$ G. Sguazzoni, ${ }^{57 \mathrm{a}}$ A. Tropiano, ${ }^{57 \mathrm{a}, 57 \mathrm{~b}}$ L. Benussi, ${ }^{58}$ S. Bianco,${ }^{58}$ F. Fabbri, ${ }^{58}$ D. Piccolo, ${ }^{58}$ P. Fabbricatore,${ }^{59 a}$ R. Musenich, ${ }^{59 a}$ S. Tosi, ${ }^{59 a, 59 b}$ A. Benaglia, ${ }^{60 a}$ F. De Guio, ${ }^{60 a, 60 b}$ L. Di Matteo, ${ }^{60 a, 60 b}$ S. Fiorendi, ${ }^{60 a, 60 b}$ S. Gennai, ${ }^{60 a}$ A. Ghezzi, ${ }^{60 a, 60 b}$ P. Govoni, ${ }^{60 a}$ M. T. Lucchini, ${ }^{60 a, c}$ S. Malvezzi, ${ }^{60 a}$ R. A. Manzoni, ${ }^{60 a, 60 b, c}$ A. Martelli, ${ }^{60 a, 60 b, c}$ A. Massironi, ${ }^{60 a, 60 b}$ D. Menasce, ${ }^{60 a}$ L. Moroni, ${ }^{60 a}$ M. Paganoni, ${ }^{60 a, 60 b}$ D. Pedrini, ${ }^{60 a}$ S. Ragazzi, ${ }^{60 a, 60 b}$ N. Redaelli, ${ }^{60 a}$ T. Tabarelli de Fatis, ${ }^{60 a, 60 b}$ S. Buontempo, ${ }^{61 \mathrm{a}}$ N. Cavallo, ${ }^{61 \mathrm{a}, 61 \mathrm{c}}$ A. De Cosa,${ }^{61 \mathrm{a}, 61 \mathrm{~b}}$ F. Fabozzi, ${ }^{61 \mathrm{a}, 61 \mathrm{c}}$ A. O. M. Iorio, ${ }^{61 \mathrm{a}, 61 \mathrm{~b}}$ L. Lista, ${ }^{61 \mathrm{a}}$ S. Meola,${ }^{61 \mathrm{a}, 61 \mathrm{~d}, \mathrm{c}}$ M. Merola,${ }^{61 \mathrm{a}}$ P. Paolucci, ${ }^{61 \mathrm{a}, \mathrm{c}}$ P. Azzi, ${ }^{62 \mathrm{a}}$ N. Bacchetta, ${ }^{62 \mathrm{a}}$ D. Bisello, ${ }^{62 a, 62 b}$ A. Branca, ${ }^{62 a, 62 b}$ R. Carlin, ${ }^{62 a, 62 b}$ P. Checchia, ${ }^{62 a}$ T. Dorigo, ${ }^{62 a}$ U. Dosselli, ${ }^{62 a}$ M. Galanti, ${ }^{62 a, 62 b, c}$ F. Gasparini, ${ }^{62 a, 62 b}$ U. Gasparini, ${ }^{62 a, 62 b}$ P. Giubilato, ${ }^{62 a, 62 b}$ A. Gozzelino, ${ }^{62 a}$ K. Kanishchev, ${ }^{62 a, 62 c}$ S. Lacaprara, ${ }^{62 a}$ I. Lazzizzera, ${ }^{62 \mathrm{a}, 62 \mathrm{c}}$ M. Margoni, ${ }^{62 \mathrm{a}, 62 \mathrm{~b}}$ A. T. Meneguzzo, ${ }^{62 \mathrm{a}, 62 \mathrm{~b}}$ M. Passaseo, ${ }^{62 \mathrm{a}}$ J. Pazzini ${ }^{62 \mathrm{a}, 62 \mathrm{~b}}$ M. Pegoraro, ${ }^{62 \mathrm{a}}$ N. Pozzobon, ${ }^{62 a, 62 b}$ P. Ronchese, ${ }^{62 a, 62 b}$ F. Simonetto, ${ }^{62 a, 62 b}$ E. Torassa, ${ }^{62 a}$ M. Tosi, ${ }^{62 a, 62 b}$ S. Ventura, ${ }^{62 a}$ P. Zotto, ${ }^{62 a, 62 b}$ A. Zucchetta, ${ }^{62 a, 62 b}$ G. Zumerle, ${ }^{62 a, 62 b}$ M. Gabusi, ${ }^{63 a, 63 b}$ S. P. Ratti, ${ }^{63 a, 63 b}$ C. Riccardi, ${ }^{63 a, 63 b}$ P. Vitulo, ${ }^{63 a, 63 b}$ M. Biasini, ${ }^{64 a, 64 b}$ G. M. Bilei, ${ }^{64 a}$ L. Fanò, ${ }^{64 a, 64 b}$ P. Lariccia, ${ }^{64 a, 64 b}$ G. Mantovani, ${ }^{64 a, 64 b}$ M. Menichelli, ${ }^{64 a}$ A. Nappi, ${ }^{64 a, 64 b, a}$ F. Romeo, ${ }^{64 a, 64 b}$ A. Saha, ${ }^{64 a}$ A. Santocchia, ${ }^{64 a, 64 b}$ A. Spiezia, ${ }^{64 a, 64 b}$ K. Androsov ${ }^{65 a, d d}$ P. Azzurri, ${ }^{65 a}$ G. Bagliesi, ${ }^{65 a}$ T. Boccali, ${ }^{65 a}$ G. Broccolo, ${ }^{65 a, 65 c}$ R. Castaldi, ${ }^{65 a}$ R. T. D’Agnolo, ${ }^{65 a, 65 c, c}$ R. Dell'Orso, ${ }^{65 a}$ F. Fiori, ${ }^{65 a, 65 c}$ L. Foà, ${ }^{65 a, 65 c}$ A. Giassi, ${ }^{65 a}$ A. Kraan, ${ }^{65 a}$ F. Ligabue, ${ }^{65 a, 65 c}$ T. Lomtadze, ${ }^{65 a}$ L. Martini, ${ }^{65 a, d d}$ A. Messineo, ${ }^{65 a, 65 b}$ F. Palla, ${ }^{65 a}$ A. Rizzi, ${ }^{65 a, 65 b}$ A. T. Serban, ${ }^{65 a}$ P. Spagnolo, ${ }^{65 a}$ P. Squillacioti, ${ }^{65 a}$ R. Tenchini, ${ }^{65 a}$ G. Tonelli, ${ }^{65 a, 65 b}$ A. Venturi, ${ }^{65 a}$ P. G. Verdini, ${ }^{65 a}$ C. Vernieri, ${ }^{65 a, 65 c}$ L. Barone, ${ }^{66 a, 66 b}$ F. Cavallari, ${ }^{66 \mathrm{a}}$ D. Del Re, ${ }^{66 a, 66 \mathrm{~b}}$ M. Diemoz, ${ }^{66 \mathrm{a}}$ M. Grassi, ${ }^{66 \mathrm{a}, 66 \mathrm{~b}, \mathrm{c}}$ E. Longo, ${ }^{66 \mathrm{a}, 66 \mathrm{~b}}$ F. Margaroli, ${ }^{66 \mathrm{6a}, 66 \mathrm{~b}}$ P. Meridiani, ${ }^{66 \mathrm{a}}$ F. Micheli, ${ }^{66 a, 66 \mathrm{~b}}$ S. Nourbakhsh ${ }^{66 \mathrm{a}, 66 \mathrm{~b}}$ G. Organtini, ${ }^{66 \mathrm{a}, 66 \mathrm{~b}}$ R. Paramatti, ${ }^{66 \mathrm{a}}$ S. Rahatlou, ${ }^{66 \mathrm{a}, 66 \mathrm{~b}}$ L. Soffi, ${ }^{66 a, 66 b}$ N. Amapane, ${ }^{67 a, 67 b}$ R. Arcidiacono, ${ }^{67 a, 67 c}$ S. Argiro, ${ }^{67 a, 67 b}$ M. Arneodo, ${ }^{67 a, 67 c}$ C. Biino, ${ }^{67 a}$ N. Cartiglia, ${ }^{67 a}$ S. Casasso, ${ }^{67 a, 67 b}$ M. Costa, ${ }^{67 a, 67 b}$ P. De Remigis, ${ }^{67 a}$ N. Demaria, ${ }^{67 a}$ C. Mariotti, ${ }^{67 a}$ S. Maselli, ${ }^{67 a}$ 
E. Migliore, ${ }^{67 a, 67 b}$ V. Monaco, ${ }^{67 a, 67 b}$ M. Musich,${ }^{67 a}$ M. M. Obertino, ${ }^{67 a, 67 c}$ N. Pastrone, ${ }^{67 a}$ M. Pelliccioni, ${ }^{67 a, c}$ A. Potenza, ${ }^{67 a, 67 b}$ A. Romero, ${ }^{67 a, 67 b}$ M. Ruspa,${ }^{67 a, 67 c}$ R. Sacchi, ${ }^{67 a, 67 b}$ A. Solano, ${ }^{67 a, 67 b}$ A. Staiano, ${ }^{67 a}$ U. Tamponi, ${ }^{67 a}$ S. Belforte, ${ }^{68 \mathrm{a}}$ V. Candelise, ${ }^{68 \mathrm{a}, 68 \mathrm{~b}} \mathrm{M}$. Casarsa ${ }^{68 \mathrm{a}}$ F. Cossutti, ${ }^{68 \mathrm{a}, \mathrm{c}}$ G. Della Ricca, ${ }^{68 \mathrm{a}, 68 \mathrm{~b}}$ B. Gobbo, ${ }^{68 \mathrm{a}}$ C. La Licata ${ }^{68 a, 68 b}$ M. Marone, ${ }^{68 a, 68 b}$ D. Montanino,${ }^{68 a, 68 b}$ A. Penzo, ${ }^{68 a}$ A. Schizzi,${ }^{68 a, 68 b}$ A. Zanetti, ${ }^{68 a}$ T. Y. Kim, ${ }^{69}$ S. K. Nam ${ }^{69}$ S. Chang, ${ }^{70}$ D. H. Kim,${ }^{70}$ G. N. Kim, ${ }^{70}$ J. E. Kim, ${ }^{70}$ D. J. Kong, ${ }^{70}$ Y. D. Oh, ${ }^{70}$ H. Park, ${ }^{70}$ D. C. Son, ${ }^{70}$ J. Y. Kim, ${ }^{71}$ Zero J. Kim, ${ }^{71}$ S. Song, ${ }^{71}$ S. Choi,${ }^{72}$ D. Gyun, ${ }^{72}$ B. Hong, ${ }^{72}$ M. Jo,${ }^{72}$ H. Kim, ${ }^{72}$ T. J. Kim, ${ }^{72}$ K. S. Lee, ${ }^{72}$ S. K. Park, ${ }^{72}$ Y. Roh,${ }^{72}$ M. Choi, ${ }^{73}$ J. H. Kim, ${ }^{73}$ C. Park, ${ }^{73}$ I. C. Park, ${ }^{73}$ S. Park, ${ }^{73}$ G. Ryu ${ }^{73}$ Y. Choi, ${ }^{74}$ Y. K. Choi, ${ }^{74}$ J. Goh, ${ }^{74}$ M. S. Kim, ${ }^{74}$ E. Kwon, ${ }^{74}$ B. Lee, ${ }^{74}$ J. Lee, ${ }^{74}$ S. Lee, ${ }^{74}$ H. Seo, ${ }^{74}$ I. Yu,${ }^{74}$ I. Grigelionis,${ }^{75}$ A. Juodagalvis,${ }^{75}$ H. Castilla-Valdez ${ }^{76}$ E. De La Cruz-Burelo, ${ }^{76}$ I. Heredia-de La Cruz, ${ }^{76, e e}$ R. Lopez-Fernandez, ${ }^{76}$

J. Martínez-Ortega, ${ }^{76}$ A. Sanchez-Hernandez,${ }^{76}$ L. M. Villasenor-Cendejas, ${ }^{76}$ S. Carrillo Moreno, ${ }^{77}$

F. Vazquez Valencia ${ }^{77}$ H. A. Salazar Ibarguen, ${ }^{78}$ E. Casimiro Linares,${ }^{79}$ A. Morelos Pineda,${ }^{79}$ M. A. Reyes-Santos, ${ }^{79}$ D. Krofcheck, ${ }^{80}$ A. J. Bell, ${ }^{81}$ P. H. Butler, ${ }^{81}$ R. Doesburg, ${ }^{81}$ S. Reucroft,${ }^{81}$ H. Silverwood, ${ }^{81}$ M. Ahmad, ${ }^{82}$ M. I. Asghar, ${ }^{82}$ J. Butt, ${ }^{82}$ H. R. Hoorani, ${ }^{82}$ S. Khalid, ${ }^{82}$ W. A. Khan,${ }^{82}$ T. Khurshid, ${ }^{82}$ S. Qazi, ${ }^{82}$ M. A. Shah, ${ }^{82}$ M. Shoaib, ${ }^{82}$ H. Bialkowska, ${ }^{83}$ B. Boimska, ${ }^{83}$ T. Frueboes, ${ }^{83}$ M. Górski, ${ }^{83}$ M. Kazana, ${ }^{83}$ K. Nawrocki, ${ }^{83}$ K. Romanowska-Rybinska, ${ }^{83}$ M. Szleper, ${ }^{83}$ G. Wrochna, ${ }^{83}$ P. Zalewski, ${ }^{83}$ G. Brona ${ }^{84}$ K. Bunkowski, ${ }^{84}$ M. Cwiok ${ }^{84}$ W. Dominik,${ }^{84}$ K. Doroba, ${ }^{84}$ A. Kalinowski, ${ }^{84}$ M. Konecki, ${ }^{84}$ J. Krolikowski, ${ }^{84}$ M. Misiura, ${ }^{84}$ W. Wolszczak, ${ }^{84}$ N. Almeida, ${ }^{85}$ P. Bargassa, ${ }^{85}$ A. David, ${ }^{85}$ P. Faccioli, ${ }^{85}$ P. G. Ferreira Parracho, ${ }^{85}$ M. Gallinaro, ${ }^{85}$

J. Rodrigues Antunes, ${ }^{85}$ J. Seixas, ${ }^{85, \mathrm{c}}$ J. Varela, ${ }^{85} \mathrm{P}$. Vischia, ${ }^{85} \mathrm{P}$. Bunin, ${ }^{86} \mathrm{M}$. Gavrilenko, ${ }^{86} \mathrm{I}$. Golutvin, ${ }^{86}$ I. Gorbunov, ${ }^{86}$ A. Kamenev, ${ }^{86}$ V. Karjavin,${ }^{86}$ V. Konoplyanikov, ${ }^{86}$ G. Kozlov, ${ }^{86}$ A. Lanev, ${ }^{86}$ A. Malakhov, ${ }^{86}$ V. Matveev, ${ }^{86}$ P. Moisenz, ${ }^{86}$ V. Palichik ${ }^{86}$ V. Perelygin, ${ }^{86}$ S. Shmatov,${ }^{86}$ N. Skatchkov ${ }^{86}$ V. Smirnov, ${ }^{86}$ A. Zarubin, ${ }^{86}$ S. Evstyukhin, ${ }^{87}$ V. Golovtsov, ${ }^{87}$ Y. Ivanov, ${ }^{87}$ V. Kim,${ }^{87}$ P. Levchenko, ${ }^{87}$ V. Murzin,${ }^{87}$ V. Oreshkin,${ }^{87}$ I. Smirnov, ${ }^{87}$ V. Sulimov, ${ }^{87}$ L. Uvarov, ${ }^{87}$ S. Vavilov, ${ }^{87}$ A. Vorobyev, ${ }^{87}$ An. Vorobyev,${ }^{87}$ Yu. Andreev, ${ }^{88}$ A. Dermenev, ${ }^{88}$ S. Gninenko, ${ }^{88}$ N. Golubev ${ }^{88}$ M. Kirsanov, ${ }^{88}$ N. Krasnikov, ${ }^{88}$ A. Pashenkov ${ }^{88}$ D. Tlisov, ${ }^{88}$ A. Toropin, ${ }^{88}$ V. Epshteyn ${ }^{89}$ M. Erofeeva, ${ }^{89}$ V. Gavrilov, ${ }^{89}$ N. Lychkovskaya, ${ }^{89}$ V. Popov, ${ }^{89}$ G. Safronov, ${ }^{89}$ S. Semenov,${ }^{89}$ A. Spiridonov, ${ }^{89}$ V. Stolin, ${ }^{89}$ E. Vlasov, ${ }^{89}$ A. Zhokin, ${ }^{89}$ V. Andreev, ${ }^{90}$ M. Azarkin,,${ }^{90}$ I. Dremin, ${ }^{90}$ M. Kirakosyan, ${ }^{90}$

A. Leonidov, ${ }^{90}$ G. Mesyats, ${ }^{90}$ S. V. Rusakov, ${ }^{90}$ A. Vinogradov, ${ }^{90}$ A. Belyaev, ${ }^{91}$ E. Boos, ${ }^{91}$ M. Dubinin,,${ }^{91, h}$ L. Dudko, ${ }^{91}$ A. Ershov, ${ }^{91}$ A. Gribushin, ${ }^{91}$ V. Klyukhin, ${ }^{91}$ O. Kodolova, ${ }^{91}$ I. Lokhtin, ${ }^{91}$ A. Markina, ${ }^{91}$ S. Obraztsov, ${ }^{91}$ S. Petrushanko, ${ }^{91}$ V. Savrin, ${ }^{91}$ A. Snigirev, ${ }^{91}$ I. Azhgirey, ${ }^{92}$ I. Bayshev,${ }^{92}$ S. Bitioukov,${ }^{92}$ V. Kachanov, ${ }^{92}$ A. Kalinin, ${ }^{92}$

D. Konstantinov, ${ }^{92}$ V. Krychkine, ${ }^{92}$ V. Petrov, ${ }^{92}$ R. Ryutin, ${ }^{92}$ A. Sobol,${ }^{92}$ L. Tourtchanovitch, ${ }^{92}$ S. Troshin, ${ }^{92}$

N. Tyurin, ${ }^{92}$ A. Uzunian, ${ }^{92}$ A. Volkov, ${ }^{92}$ P. Adzic, ${ }^{93, f f}$ M. Ekmedzic, ${ }^{93}$ D. Krpic,${ }^{93, f f}$ J. Milosevic, ${ }^{93}$ M. Aguilar-Benitez, ${ }^{94}$ J. Alcaraz Maestre, ${ }^{94}$ C. Battilana, ${ }^{94}$ E. Calvo, ${ }^{94}$ M. Cerrada, ${ }^{94}$ M. Chamizo Llatas, ${ }^{94, c}$ N. Colino, ${ }^{94}$ B. De La Cruz, ${ }^{94}$ A. Delgado Peris,${ }^{94}$ D. Domínguez Vázquez, ${ }^{94}$ C. Fernandez Bedoya,${ }^{94}$ J. P. Fernández Ramos, ${ }^{94}$ A. Ferrando, ${ }^{94}$ J. Flix, ${ }^{94}$ M. C. Fouz,${ }^{94}$ P. Garcia-Abia, ${ }^{94}$ O. Gonzalez Lopez,${ }^{94}$ S. Goy Lopez, ${ }^{94}$ J. M. Hernandez, ${ }^{94}$ M. I. Josa, ${ }^{94}$ G. Merino, ${ }^{94}$ E. Navarro De Martino, ${ }^{94}$ J. Puerta Pelayo, ${ }^{94}$ A. Quintario Olmeda, ${ }^{94}$ I. Redondo, ${ }^{94}$ L. Romero, ${ }^{94}$ J. Santaolalla, ${ }^{94}$ M. S. Soares,${ }^{94}$ C. Willmott, ${ }^{94}$ C. Albajar, ${ }^{95}$ J. F. de Trocóniz, ${ }^{95}$ H. Brun, ${ }^{96}$ J. Cuevas, ${ }^{96}$ J. Fernandez Menendez, ${ }^{96}$ S. Folgueras,${ }^{96}$ I. Gonzalez Caballero, ${ }^{96}$ L. Lloret Iglesias, ${ }^{96}$ J. Piedra Gomez, ${ }^{96}$ J. A. Brochero Cifuentes, ${ }^{97}$ I. J. Cabrillo, ${ }^{97}$ A. Calderon, ${ }^{97}$ S. H. Chuang, ${ }^{97}$ J. Duarte Campderros, ${ }^{97}$ M. Fernandez,${ }^{97}$ G. Gomez, ${ }^{97}$ J. Gonzalez Sanchez, ${ }^{97}$ A. Graziano, ${ }^{97}$ C. Jorda, ${ }^{97}$ A. Lopez Virto, ${ }^{97}$ J. Marco, ${ }^{97}$ R. Marco,${ }^{97}$ C. Martinez Rivero, ${ }^{97}$ F. Matorras,${ }^{97}$ F. J. Munoz Sanchez,${ }^{97}$ T. Rodrigo, ${ }^{97}$ A. Y. Rodríguez-Marrero, ${ }^{97}$ A. Ruiz-Jimeno, ${ }^{97}$ L. Scodellaro, ${ }^{97}$ I. Vila, ${ }^{97}$ R. Vilar Cortabitarte, ${ }^{97}$ D. Abbaneo, ${ }^{98}$ E. Auffray, ${ }^{98}$ G. Auzinger, ${ }^{98}$ M. Bachtis, ${ }^{98}$ P. Baillon, ${ }^{98}$ A. H. Ball, ${ }^{98}$ D. Barney, ${ }^{98}$ J. Bendavid, ${ }^{98}$ J. F. Benitez,${ }^{98}$ C. Bernet,${ }^{98, i}$ G. Bianchi, ${ }^{98}$ P. Bloch,${ }^{98}$ A. Bocci, ${ }^{98}$ A. Bonato, ${ }^{98}$ O. Bondu, ${ }^{98}$ C. Botta,${ }^{98}$ H. Breuker,${ }^{98}$ T. Camporesi, ${ }^{98}$ G. Cerminara, ${ }^{98}$ T. Christiansen, ${ }^{98}$ J. A. Coarasa Perez, ${ }^{98}$ S. Colafranceschi, ${ }^{98, g g}$ D. d'Enterria, ${ }^{98}$ A. Dabrowski, ${ }^{98}$ A. De Roeck, ${ }^{98}$ S. De Visscher,${ }^{98}$ S. Di Guida, ${ }^{98}$ M. Dobson, ${ }^{98}$ N. Dupont-Sagorin, ${ }^{98}$

A. Elliott-Peisert, ${ }^{98}$ J. Eugster, ${ }^{98}$ W. Funk, ${ }^{98}$ G. Georgiou, ${ }^{98}$ M. Giffels, ${ }^{98}$ D. Gigi, ${ }^{98}$ K. Gill, ${ }^{98}$ D. Giordano, ${ }^{98}$ M. Girone, ${ }^{98}$ M. Giunta, ${ }^{98}$ F. Glege,${ }^{98}$ R. Gomez-Reino Garrido, ${ }^{98}$ S. Gowdy, ${ }^{98}$ R. Guida,${ }^{98}$ J. Hammer,${ }^{98}$ M. Hansen,${ }^{98}$ P. Harris, ${ }^{98}$ C. Hartl, ${ }^{98}$ B. Hegner ${ }^{98}$ A. Hinzmann, ${ }^{98}$ V. Innocente, ${ }^{98}$ P. Janot,${ }^{98}$ E. Karavakis, ${ }^{98}$ K. Kousouris, ${ }^{98}$ K. Krajczar, ${ }^{98}$ P. Lecoq,${ }^{98}$ Y.-J. Lee, ${ }^{98}$ C. Lourenço, ${ }^{98}$ N. Magini, ${ }^{98}$ M. Malberti, ${ }^{98}$ L. Malgeri, ${ }^{98}$ M. Mannelli, ${ }^{98}$ L. Masetti, ${ }^{98}$ F. Meijers, ${ }^{98}$ S. Mersi,${ }^{98}$ E. Meschi,${ }^{98}$ R. Moser,${ }^{98}$ M. Mulders,${ }^{98}$ P. Musella, ${ }^{98}$ E. Nesvold, ${ }^{98}$ L. Orsini, ${ }^{98}$ E. Palencia Cortezon, ${ }^{98}$ E. Perez, ${ }^{98}$ L. Perrozzi, ${ }^{98}$ A. Petrilli, ${ }^{98}$ A. Pfeiffer, ${ }^{98}$ M. Pierini, ${ }^{98}$ M. Pimiä, ${ }^{98}$ D. Piparo, ${ }^{98}$ M. Plagge, ${ }^{98}$ G. Polese,${ }^{98}$ L. Quertenmont, ${ }^{98}$ A. Racz,${ }^{98}$ W. Reece, ${ }^{98}$ G. Rolandi,,${ }^{98 h}$ 
C. Rovelli, ${ }^{98, \text { ii }}$ M. Rovere, ${ }^{98}$ H. Sakulin, ${ }^{98}$ F. Santanastasio, ${ }^{98}$ C. Schäfer,${ }^{98}$ C. Schwick, ${ }^{98}$ I. Segoni, ${ }^{98}$ S. Sekmen, ${ }^{98}$ A. Sharma, ${ }^{98}$ P. Siegrist,${ }^{98}$ P. Silva,${ }^{98}$ M. Simon, ${ }^{98}$ P. Sphicas, ${ }^{98, j j}$ D. Spiga,${ }^{98}$ M. Stoye, ${ }^{98}$ A. Tsirou, ${ }^{98}$ G. I. Veres, ${ }^{98, v}$ J. R. Vlimant, ${ }^{98}$ H. K. Wöhri, ${ }^{98}$ S. D. Worm, ${ }^{98, k k}$ W. D. Zeuner,${ }^{98}$ W. Bertl, ${ }^{99}$ K. Deiters, ${ }^{99}$ W. Erdmann, ${ }^{99}$ K. Gabathuler, ${ }^{99}$ R. Horisberger, ${ }^{99}$ Q. Ingram, ${ }^{99}$ H. C. Kaestli, ${ }^{99}$ S. König, ${ }^{99}$ D. Kotlinski, ${ }^{99}$ U. Langenegger, ${ }^{99}$ D. Renker ${ }^{99}$ T. Rohe,${ }^{99}$ F. Bachmair, ${ }^{100}$ L. Bäni, ${ }^{100}$ P. Bortignon, ${ }^{100}$ M. A. Buchmann, ${ }^{100}$ B. Casal, ${ }^{100}$ N. Chanon, ${ }^{100}$ A. Deisher, ${ }^{100}$ G. Dissertori, ${ }^{100}$ M. Dittmar, ${ }^{100}$ M. Donegà, ${ }^{100}$ M. Dünser, ${ }^{100}$ P. Eller, ${ }^{100}$ K. Freudenreich, ${ }^{100}$ C. Grab, ${ }^{100}$ D. Hits, ${ }^{100}$ P. Lecomte, ${ }^{100}$ W. Lustermann, ${ }^{100}$ A. C. Marini, ${ }^{100}$ P. Martinez Ruiz del Arbol, ${ }^{100}$ N. Mohr, ${ }^{100}$ F. Moortgat, ${ }^{100}$ C. Nägeli, ${ }^{100,11}$ P. Nef, ${ }^{100}$ F. Nessi-Tedaldi, ${ }^{100}$ F. Pandolfi, ${ }^{100}$ L. Pape, ${ }^{100}$ F. Pauss,${ }^{100}$ M. Peruzzi, ${ }^{100}$ F. J. Ronga, ${ }^{100}$ M. Rossini, ${ }^{100}$ L. Sala, ${ }^{100}$ A. K. Sanchez, ${ }^{100}$ A. Starodumov, ${ }^{100, m m}$ B. Stieger, ${ }^{100}$ M. Takahashi, ${ }^{100}$

L. Tauscher, ${ }^{100, a}$ A. Thea, ${ }^{100}$ K. Theofilatos, ${ }^{100}$ D. Treille, ${ }^{100}$ C. Urscheler, ${ }^{100}$ R. Wallny, ${ }^{100}$ H. A. Weber,${ }^{100}$ C. Amsler, ${ }^{101, n n}$ V. Chiochia, ${ }^{101}$ C. Favaro, ${ }^{101}$ M. Ivova Rikova, ${ }^{101}$ B. Kilminster, ${ }^{101}$ B. Millan Mejias,${ }^{101}$ P. Otiougova, ${ }^{101}$ P. Robmann, ${ }^{101}$ H. Snoek, ${ }^{101}$ S. Taroni, ${ }^{101}$ S. Tupputi, ${ }^{101}$ M. Verzetti, ${ }^{101}$ M. Cardaci, ${ }^{102}$ K. H. Chen, ${ }^{102}$ C. Ferro, ${ }^{102}$ C. M. Kuo, ${ }^{102}$ S. W. Li, ${ }^{102}$ W. Lin, ${ }^{102}$ Y. J. Lu, ${ }^{102}$ R. Volpe,${ }^{102}$ S. S. Yu, ${ }^{102}$ P. Bartalini, ${ }^{103}$ P. Chang, ${ }^{103}$ Y. H. Chang, ${ }^{103}$ Y. W. Chang, ${ }^{103}$ Y. Chao, ${ }^{103}$ K. F. Chen, ${ }^{103}$ C. Dietz, ${ }^{103}$ U. Grundler, ${ }^{103}$ W.-S. Hou, ${ }^{103}$ Y. Hsiung, ${ }^{103}$ K. Y. Kao, ${ }^{103}$ Y. J. Lei, ${ }^{103}$ R.-S. Lu, ${ }^{103}$ D. Majumder, ${ }^{103}$ E. Petrakou, ${ }^{103}$ X. Shi, ${ }^{103}$ J. G. Shiu, ${ }^{103}$

Y. M. Tzeng, ${ }^{103}$ M. Wang, ${ }^{103}$ B. Asavapibhop, ${ }^{104}$ N. Suwonjandee,,${ }^{104}$ A. Adiguzel,,${ }^{105}$ M. N. Bakirci, ${ }^{105, o o}$ S. Cerci, ${ }^{105, p p}$ C. Dozen, ${ }^{105}$ I. Dumanoglu, ${ }^{105}$ E. Eskut, ${ }^{105}$ S. Girgis, ${ }^{105}$ G. Gokbulut, ${ }^{105}$ E. Gurpinar, ${ }^{105}$ I. Hos, ${ }^{105}$ E. E. Kangal, ${ }^{105}$ A. Kayis Topaksu, ${ }^{105}$ G. Onengut, ${ }^{105, q q}$ K. Ozdemir, ${ }^{105}$ S. Ozturk, ${ }^{105, \text { oo }}$ A. Polatoz, ${ }^{105}$ K. Sogut, ${ }^{105, r r}$ D. Sunar Cerci, ${ }^{105, p p}$ B. Tali, ${ }^{105, p p}$ H. Topakli, ${ }^{105,00}$ M. Vergili, ${ }^{105}$ I. V. Akin, ${ }^{106}$ T. Aliev, ${ }^{106}$ B. Bilin, ${ }^{106}$ S. Bilmis, ${ }^{106}$ M. Deniz, ${ }^{106}$ H. Gamsizkan, ${ }^{106}$ A. M. Guler, ${ }^{106}$ G. Karapinar, ${ }^{106, s s}$ K. Ocalan, ${ }^{106}$ A. Ozpineci, ${ }^{106}$ M. Serin, ${ }^{106}$ R. Sever, ${ }^{106}$ U. E. Surat, ${ }^{106}$ M. Yalvac, ${ }^{106}$ M. Zeyrek, ${ }^{106}$ E. Gülmez, ${ }^{107}$ B. Isildak, ${ }^{107, \text { tt }}$ M. Kaya, ${ }^{107, \text { uu }}$ O. Kaya, ${ }^{107, \text { uu }}$ S. Ozkorucuklu, ${ }^{107, \mathrm{vv}}$ N. Sonmez, ${ }^{107, \mathrm{ww}}$ H. Bahtiyar, ${ }^{108, \mathrm{xx}}$ E. Barlas, ${ }^{108}$ K. Cankocak, ${ }^{108}$ Y. O. Günaydin, ${ }^{108, y y}$ F. I. Vardarl $1,{ }^{108}$ M. Yücel,${ }^{108}$ L. Levchuk, ${ }^{109}$ P. Sorokin, ${ }^{109}$ J. J. Brooke, ${ }^{110}$ E. Clement, ${ }^{110}$ D. Cussans, ${ }^{110}$ H. Flacher, ${ }^{10}$ R. Frazier, ${ }^{110}$ J. Goldstein, ${ }^{110}$ M. Grimes, ${ }^{110}$ G. P. Heath, ${ }^{110}$ H. F. Heath, ${ }^{110}$ L. Kreczko, ${ }^{110}$ S. Metson, ${ }^{110}$ D. M. Newbold, ${ }^{110, k \mathrm{k}}$ K. Nirunpong, ${ }^{110}$ A. Poll, ${ }^{110}$ S. Senkin, ${ }^{110}$ V. J. Smith, ${ }^{110}$ T. Williams, ${ }^{110}$ L. Basso, ${ }^{111, z z}$ K. W. Bell, ${ }^{111}$ A. Belyaev, ${ }^{111, z z}$ C. Brew, ${ }^{111}$ R. M. Brown, ${ }^{111}$ D. J. A. Cockerill, ${ }^{111}$ J. A. Coughlan, ${ }^{111}$ K. Harder, ${ }^{111}$ S. Harper, ${ }^{111}$ J. Jackson, ${ }^{111}$ E. Olaiya, ${ }^{111}$ D. Petyt,,${ }^{111}$ B. C. Radburn-Smith, ${ }^{111}$

C. H. Shepherd-Themistocleous, ${ }^{111}$ I. R. Tomalin, ${ }^{111}$ W. J. Womersley, ${ }^{111}$ R. Bainbridge,${ }^{112}$ O. Buchmuller, ${ }^{112}$ D. Burton, ${ }^{112}$ D. Colling, ${ }^{112}$ N. Cripps, ${ }^{112}$ M. Cutajar, ${ }^{112}$ P. Dauncey, ${ }^{112}$ G. Davies, ${ }^{112}$ M. Della Negra, ${ }^{112}$ W. Ferguson, ${ }^{112}$ J. Fulcher, ${ }^{112}$ D. Futyan, ${ }^{112}$ A. Gilbert, ${ }^{112}$ A. Guneratne Bryer, ${ }^{112}$ G. Hall, ${ }^{112}$ Z. Hatherell, ${ }^{112}$ J. Hays, ${ }^{112}$ G. Iles, ${ }^{112}$ M. Jarvis, ${ }^{112}$ G. Karapostoli, ${ }^{112}$ M. Kenzie, ${ }^{112}$ R. Lane, ${ }^{112}$ R. Lucas, ${ }^{112, k k}$ L. Lyons, ${ }^{112}$ A.-M. Magnan, ${ }^{112}$ J. Marrouche, ${ }^{112}$ B. Mathias, ${ }^{112}$ R. Nandi, ${ }^{112}$ J. Nash, ${ }^{112}$ A. Nikitenko, ${ }^{112, m m}$ J. Pela, ${ }^{112}$ M. Pesaresi, ${ }^{112}$ K. Petridis, ${ }^{112}$ M. Pioppi, ${ }^{112 \text {,aaa }}$ D. M. Raymond, ${ }^{112}$ S. Rogerson,,${ }^{112}$ A. Rose,${ }^{112}$ C. Seez,${ }^{112}$ P. Sharp,${ }^{112, a}$ A. Sparrow, ${ }^{112}$ A. Tapper, ${ }^{112}$ M. Vazquez Acosta, ${ }^{112}$ T. Virdee,${ }^{112}$ S. Wakefield,${ }^{112}$ N. Wardle, ${ }^{112}$ T. Whyntie, ${ }^{112}$ M. Chadwick, ${ }^{113}$ J. E. Cole, ${ }^{113}$ P. R. Hobson, ${ }^{113}$ A. Khan, ${ }^{113}$ P. Kyberd,${ }^{113}$ D. Leggat,${ }^{113}$ D. Leslie,${ }^{113}$ W. Martin, ${ }^{113}$ I. D. Reid,${ }^{113}$ P. Symonds, ${ }^{113}$ L. Teodorescu, ${ }^{113}$ M. Turner, ${ }^{113}$ J. Dittmann, ${ }^{114}$ K. Hatakeyama, ${ }^{114}$

A. Kasmi, ${ }^{114}$ H. Liu, ${ }^{114}$ T. Scarborough, ${ }^{114}$ O. Charaf, ${ }^{115}$ S. I. Cooper,,${ }^{15}$ C. Henderson,,${ }^{115}$ P. Rumerio,,${ }^{115}$ A. Avetisyan, ${ }^{116}$ T. Bose,${ }^{116}$ C. Fantasia, ${ }^{116}$ A. Heister ${ }^{116}$ P. Lawson, ${ }^{116}$ D. Lazic, ${ }^{116}$ J. Rohlf, ${ }^{116}$ D. Sperka, ${ }^{116}$ J. St. John, ${ }^{116}$ L. Sulak, ${ }^{116}$ J. Alimena,${ }^{117}$ S. Bhattacharya, ${ }^{117}$ G. Christopher, ${ }^{117}$ D. Cutts, ${ }^{117}$ Z. Demiragli, ${ }^{117}$ A. Ferapontov, ${ }^{117}$ A. Garabedian, ${ }^{117}$ U. Heintz, ${ }^{117}$ G. Kukartsev, ${ }^{117}$ E. Laird, ${ }^{117}$ G. Landsberg, ${ }^{117}$ M. Luk, ${ }^{117}$ M. Narain, ${ }^{117}$ M. Segala, ${ }^{117}$ T. Sinthuprasith, ${ }^{117}$ T. Speer, ${ }^{117}$ R. Breedon, ${ }^{118}$ G. Breto, ${ }^{118}$

M. Calderon De La Barca Sanchez, ${ }^{118}$ S. Chauhan, ${ }^{118}$ M. Chertok, ${ }^{118}$ J. Conway, ${ }^{118}$ R. Conway, ${ }^{118}$ P. T. Cox, ${ }^{118}$ R. Erbacher, ${ }^{18}$ M. Gardner, ${ }^{18}$ R. Houtz, ${ }^{118}$ W. Ko, ${ }^{18}$ A. Kopecky, ${ }^{118}$ R. Lander, ${ }^{118}$ O. Mall, ${ }^{118}$ T. Miceli, ${ }^{118}$ R. Nelson, ${ }^{118}$ D. Pellett, ${ }^{118}$ F. Ricci-Tam, ${ }^{118}$ B. Rutherford, ${ }^{118}$ M. Searle, ${ }^{118}$ J. Smith, ${ }^{118}$ M. Squires, ${ }^{118}$ M. Tripathi, ${ }^{118}$ S. Wilbur, ${ }^{118}$ R. Yohay, ${ }^{118}$ V. Andreev, ${ }^{119}$ D. Cline,,${ }^{119}$ R. Cousins, ${ }^{119}$ S. Erhan, ${ }^{119}$ P. Everaerts, ${ }^{119}$ C. Farrell, ${ }^{119}$ M. Felcini, ${ }^{119}$ J. Hauser,${ }^{119}$ M. Ignatenko, ${ }^{119}$ C. Jarvis, ${ }^{119}$ G. Rakness, ${ }^{119}$ P. Schlein, ${ }^{119, a}$ E. Takasugi, ${ }^{119}$ P. Traczyk, ${ }^{119}$ V. Valuev, ${ }^{119}$ M. Weber, ${ }^{119}$ J. Babb, ${ }^{120}$ R. Clare, ${ }^{120}$ M.E. Dinardo, ${ }^{120}$ J. Ellison, ${ }^{120}$ J. W. Gary, ${ }^{120}$ G. Hanson, ${ }^{120}$ H. Liu, ${ }^{120}$ O. R. Long, ${ }^{120}$ A. Luthra, ${ }^{120}$ H. Nguyen, ${ }^{120}$ S. Paramesvaran, ${ }^{120}$ J. Sturdy, ${ }^{120}$

S. Sumowidagdo, ${ }^{120}$ R. Wilken, ${ }^{120}$ S. Wimpenny, ${ }^{120}$ W. Andrews, ${ }^{121}$ J. G. Branson, ${ }^{121}$ G. B. Cerati, ${ }^{121}$ S. Cittolin, ${ }^{121}$ D. Evans, ${ }^{121}$ A. Holzner, ${ }^{121}$ R. Kelley, ${ }^{121}$ M. Lebourgeois, ${ }^{121}$ J. Letts, ${ }^{121}$ I. Macneill, ${ }^{121}$ B. Mangano, ${ }^{121}$ S. Padhi, ${ }^{121}$ C. Palmer, ${ }^{121}$ G. Petrucciani, ${ }^{121}$ M. Pieri, ${ }^{121}$ M. Sani, ${ }^{121}$ V. Sharma, ${ }^{121}$ S. Simon, ${ }^{121}$ E. Sudano, ${ }^{121}$ M. Tadel,${ }^{121}$ 
Y. Tu, ${ }^{121}$ A. Vartak, ${ }^{121}$ S. Wasserbaech, ${ }^{121, b b b}$ F. Würthwein, ${ }^{121}$ A. Yagil, ${ }^{121}$ J. Yoo, ${ }^{121}$ D. Barge, ${ }^{122}$ R. Bellan, ${ }^{122}$

C. Campagnari, ${ }^{122}$ M. D'Alfonso, ${ }^{122}$ T. Danielson, ${ }^{122}$ K. Flowers, ${ }^{122}$ P. Geffert, ${ }^{122}$ C. George, ${ }^{122}$ F. Golf, ${ }^{122}$ J. Incandela, ${ }^{122}$ C. Justus, ${ }^{122}$ P. Kalavase, ${ }^{122}$ D. Kovalskyi, ${ }^{122}$ V. Krutelyov, ${ }^{122}$ S. Lowette, ${ }^{122}$ R. Magaña Villalba, ${ }^{122}$ N. Mccoll, ${ }^{122}$ V. Pavlunin, ${ }^{122}$ J. Ribnik, ${ }^{122}$ J. Richman, ${ }^{122}$ R. Rossin, ${ }^{122}$ D. Stuart, ${ }^{122}$ W. To, ${ }^{122}$ C. West, ${ }^{122}$ A. Apresyan, ${ }^{123}$ A. Bornheim, ${ }^{123}$ J. Bunn, ${ }^{123}$ Y. Chen, ${ }^{123}$ E. Di Marco, ${ }^{123}$ J. Duarte, ${ }^{123}$ D. Kcira, ${ }^{123}$ Y. Ma, ${ }^{123}$ A. Mott, ${ }^{123}$ H. B. Newman, ${ }^{123}$ C. Rogan, ${ }^{123}$ M. Spiropulu, ${ }^{123}$ V. Timciuc, ${ }^{123}$ J. Veverka, ${ }^{123}$ R. Wilkinson, ${ }^{123}$ S. Xie, ${ }^{123}$ Y. Yang, ${ }^{123}$ R. Y. Zhu, ${ }^{123}$ V. Azzolini, ${ }^{124}$ A. Calamba,${ }^{124}$ R. Carroll, ${ }^{124}$ T. Ferguson, ${ }^{124}$ Y. Iiyama, ${ }^{124}$ D. W. Jang, ${ }^{124}$ Y. F. Liu, ${ }^{124}$ M. Paulini, ${ }^{124}$ J. Russ, ${ }^{124}$ H. Vogel,${ }^{124}$ I. Vorobiev, ${ }^{124}$ J. P. Cumalat,${ }^{125}$ B. R. Drell, ${ }^{125}$ W. T. Ford, ${ }^{125}$ A. Gaz, ${ }^{125}$ E. Luiggi Lopez, ${ }^{125}$ U. Nauenberg, ${ }^{125}$ J. G. Smith, ${ }^{125}$ K. Stenson, ${ }^{125}$ K. A. Ulmer, ${ }^{125}$

S. R. Wagner, ${ }^{125}$ J. Alexander, ${ }^{126}$ A. Chatterjee, ${ }^{126}$ N. Eggert, ${ }^{126}$ L. K. Gibbons, ${ }^{126}$ W. Hopkins, ${ }^{126}$

A. Khukhunaishvili, ${ }^{126}$ B. Kreis, ${ }^{126}$ N. Mirman, ${ }^{126}$ G. Nicolas Kaufman, ${ }^{126}$ J. R. Patterson, ${ }^{126}$ A. Ryd, ${ }^{126}$ E. Salvati, ${ }^{126}$ W. Sun, ${ }^{126}$ W. D. Teo, ${ }^{126}$ J. Thom,${ }^{126}$ J. Thompson, ${ }^{126}$ J. Tucker, ${ }^{126}$ Y. Weng, ${ }^{126}$ L. Winstrom, ${ }^{126}$ P. Wittich, ${ }^{126}$ D. Winn, ${ }^{127}$ S. Abdullin, ${ }^{128}$ M. Albrow, ${ }^{128}$ J. Anderson, ${ }^{128}$ G. Apollinari, ${ }^{128}$ L. A. T. Bauerdick, ${ }^{128}$ A. Beretvas, ${ }^{128}$ J. Berryhill, ${ }^{128}$ P. C. Bhat, ${ }^{128}$ K. Burkett, ${ }^{128}$ J. N. Butler,${ }^{128}$ V. Chetluru, ${ }^{128}$ H. W. K. Cheung, ${ }^{128}$ F. Chlebana, ${ }^{128}$ S. Cihangir, ${ }^{128}$ V. D. Elvira, ${ }^{128}$ I. Fisk, ${ }^{128}$ J. Freeman, ${ }^{128}$ Y. Gao, ${ }^{128}$ E. Gottschalk, ${ }^{128}$ L. Gray, ${ }^{128}$ D. Green, ${ }^{128}$ O. Gutsche, ${ }^{128}$ D. Hare, ${ }^{128}$ R. M. Harris, ${ }^{128}$ J. Hirschauer, ${ }^{128}$ B. Hooberman, ${ }^{128}$ S. Jindariani, ${ }^{128}$ M. Johnson, ${ }^{128}$ U. Joshi, ${ }^{128}$ B. Klima, ${ }^{128}$ S. Kunori, ${ }^{128}$ S. Kwan, ${ }^{128}$ C. Leonidopoulos, ${ }^{128, c c c}$ J. Linacre, ${ }^{128}$ D. Lincoln, ${ }^{128}$ R. Lipton, ${ }^{128}$ J. Lykken, ${ }^{128}$ K. Maeshima, ${ }^{128}$ J. M. Marraffino, ${ }^{128}$ V. I. Martinez Outschoorn, ${ }^{128}$ S. Maruyama, ${ }^{128}$ D. Mason, ${ }^{128}$ P. McBride, ${ }^{128}$ K. Mishra, ${ }^{128}$ S. Mrenna, ${ }^{128}$ Y. Musienko, ${ }^{128, \text { ddd }}$

C. Newman-Holmes, ${ }^{128}$ V. O’Dell, ${ }^{128}$ O. Prokofyev, ${ }^{128}$ N. Ratnikova, ${ }^{128}$ E. Sexton-Kennedy, ${ }^{128}$ S. Sharma, ${ }^{128}$ W. J. Spalding, ${ }^{128}$ L. Spiegel, ${ }^{128}$ L. Taylor, ${ }^{128}$ S. Tkaczyk, ${ }^{128}$ N. V. Tran, ${ }^{128}$ L. Uplegger, ${ }^{128}$ E. W. Vaandering, ${ }^{128}$

R. Vidal,${ }^{128}$ J. Whitmore, ${ }^{128}$ W. Wu, ${ }^{128}$ F. Yang, ${ }^{128}$ J. C. Yun, ${ }^{128}$ D. Acosta, ${ }^{129}$ P. Avery,${ }^{129}$ D. Bourilkov, ${ }^{129}$ M. Chen, ${ }^{129}$ T. Cheng, ${ }^{129}$ S. Das, ${ }^{129}$ M. De Gruttola, ${ }^{129}$ G. P. Di Giovanni, ${ }^{129}$ D. Dobur, ${ }^{129}$ A. Drozdetskiy, ${ }^{129}$ R. D. Field, ${ }^{129}$ M. Fisher, ${ }^{129}$ Y. Fu, ${ }^{129}$ I. K. Furic,${ }^{129}$ J. Hugon, ${ }^{129}$ B. Kim,${ }^{129}$ J. Konigsberg, ${ }^{129}$ A. Korytov, ${ }^{129}$

A. Kropivnitskaya, ${ }^{129}$ T. Kypreos, ${ }^{129}$ J. F. Low, ${ }^{129}$ K. Matchev, ${ }^{129}$ P. Milenovic, ${ }^{129, \text { eee }}$ G. Mitselmakher, ${ }^{129}$ L. Muniz, ${ }^{129}$ R. Remington, ${ }^{129}$ A. Rinkevicius, ${ }^{129}$ N. Skhirtladze, ${ }^{129}$ M. Snowball, ${ }^{129}$ J. Yelton, ${ }^{129}$ M. Zakaria, ${ }^{129}$ V. Gaultney, ${ }^{130}$ S. Hewamanage, ${ }^{130}$ L. M. Lebolo, ${ }^{130}$ S. Linn, ${ }^{130}$ P. Markowitz, ${ }^{130}$ G. Martinez, ${ }^{130}$ J. L. Rodriguez, ${ }^{130}$ T. Adams, ${ }^{131}$ A. Askew, ${ }^{131}$ J. Bochenek, ${ }^{131}$ J. Chen, ${ }^{131}$ B. Diamond, ${ }^{131}$ S. V. Gleyzer, ${ }^{131}$ J. Haas, ${ }^{131}$ S. Hagopian, ${ }^{131}$

V. Hagopian, ${ }^{131}$ K. F. Johnson, ${ }^{131}$ H. Prosper, ${ }^{131}$ V. Veeraraghavan, ${ }^{131}$ M. Weinberg, ${ }^{131}$ M. M. Baarmand, ${ }^{132}$

B. Dorney, ${ }^{132}$ M. Hohlmann, ${ }^{132}$ H. Kalakhety, ${ }^{132}$ F. Yumiceva,${ }^{132}$ M. R. Adams, ${ }^{133}$ L. Apanasevich, ${ }^{133}$

V. E. Bazterra, ${ }^{133}$ R. R. Betts, ${ }^{133}$ I. Bucinskaite, ${ }^{133}$ J. Callner ${ }^{133}$ R. Cavanaugh, ${ }^{133}$ O. Evdokimov, ${ }^{133}$ L. Gauthier, ${ }^{133}$

C. E. Gerber, ${ }^{133}$ D. J. Hofman, ${ }^{133}$ S. Khalatyan, ${ }^{133}$ P. Kurt, ${ }^{133}$ F. Lacroix, ${ }^{133}$ D. H. Moon, ${ }^{133}$ C. O'Brien, ${ }^{133}$

C. Silkworth, ${ }^{133}$ D. Strom, ${ }^{133}$ P. Turner, ${ }^{133}$ N. Varelas, ${ }^{133}$ U. Akgun, ${ }^{134}$ E. A. Albayrak,${ }^{134, x x}$ B. Bilki, ${ }^{134, f f f}$ W. Clarida, ${ }^{134}$ K. Dilsiz, ${ }^{134}$ F. Duru, ${ }^{134}$ S. Griffiths, ${ }^{134}$ J.-P. Merlo, ${ }^{134}$ H. Mermerkaya, ${ }^{134, \text { ggg }}$ A. Mestvirishvili, ${ }^{134}$ A. Moeller, ${ }^{134}$ J. Nachtman, ${ }^{134}$ C. R. Newsom, ${ }^{134}$ H. Ogul, ${ }^{134}$ Y. Onel, ${ }^{134}$ F. Ozok, ${ }^{134, x x}$ S. Sen, ${ }^{134}$ P. Tan, ${ }^{134}$ E. Tiras, ${ }^{134}$ J. Wetzel, ${ }^{134}$ T. Yetkin, ${ }^{134, \text { hhh }}$ K. Yi, ${ }^{134}$ B. A. Barnett,,${ }^{135}$ B. Blumenfeld, ${ }^{135}$ S. Bolognesi, ${ }^{135}$ D. Fehling, ${ }^{135}$ G. Giurgiu, ${ }^{135}$ A. V. Gritsan, ${ }^{135}$ Z. J. Guo, ${ }^{135}$ G. Hu, ${ }^{135}$ P. Maksimovic, ${ }^{135}$ M. Swartz, ${ }^{135}$ A. Whitbeck, ${ }^{135}$ P. Baringer, ${ }^{136}$ A. Bean, ${ }^{136}$ G. Benelli, ${ }^{136}$ R. P. Kenny III, ${ }^{136}$ M. Murray, ${ }^{136}$ D. Noonan, ${ }^{136}$ S. Sanders, ${ }^{136}$ R. Stringer, ${ }^{136}$ J. S. Wood, ${ }^{136}$ A. F. Barfuss, ${ }^{137}$ I. Chakaberia, ${ }^{137}$ A. Ivanov, ${ }^{137}$ S. Khalil, ${ }^{137}$ M. Makouski, ${ }^{137}$ Y. Maravin, ${ }^{137}$ S. Shrestha, ${ }^{137}$ I. Svintradze, ${ }^{137}$ J. Gronberg, ${ }^{138}$ D. Lange, ${ }^{138}$ F. Rebassoo, ${ }^{138}$ D. Wright, ${ }^{138}$ A. Baden, ${ }^{139}$ B. Calvert, ${ }^{139}$ S. C. Eno, ${ }^{139}$ J. A. Gomez, ${ }^{139}$ N. J. Hadley, ${ }^{139}$ R. G. Kellogg, ${ }^{139}$ T. Kolberg, ${ }^{139}$ Y. Lu, ${ }^{139}$ M. Marionneau, ${ }^{139}$ A. C. Mignerey, ${ }^{139}$ K. Pedro, ${ }^{139}$ A. Peterman, ${ }^{139}$ A. Skuja, ${ }^{139}$ J. Temple, ${ }^{139}$ M. B. Tonjes, ${ }^{139}$ S. C. Tonwar, ${ }^{139}$ A. Apyan, ${ }^{140}$ G. Bauer, ${ }^{140}$ W. Busza, ${ }^{140}$ E. Butz, ${ }^{140}$ I. A. Cali, ${ }^{140}$ M. Chan, ${ }^{140}$ V. Dutta, ${ }^{140}$ G. Gomez Ceballos, ${ }^{140}$ M. Goncharov, ${ }^{140}$ Y. Kim, ${ }^{140}$ M. Klute, ${ }^{140}$ Y. S. Lai, ${ }^{140}$ A. Levin, ${ }^{140}$ P. D. Luckey, ${ }^{140}$ T. Ma, ${ }^{140}$ S. Nahn, ${ }^{140}$ C. Paus,${ }^{140}$ D. Ralph,${ }^{140}$ C. Roland, ${ }^{140}$ G. Roland, ${ }^{140}$ G. S. F. Stephans, ${ }^{140}$ F. Stöckli, ${ }^{140}$ K. Sumorok, ${ }^{140}$ K. Sung, ${ }^{140}$ D. Velicanu, ${ }^{140}$ R. Wolf, ${ }^{140}$ B. Wyslouch, ${ }^{140}$ M. Yang, ${ }^{140}$ Y. Yilmaz, ${ }^{140}$ A. S. Yoon, ${ }^{140}$ M. Zanetti, ${ }^{140}$ V. Zhukova, ${ }^{140}$ B. Dahmes, ${ }^{141}$ A. De Benedetti, ${ }^{141}$ G. Franzoni, ${ }^{141}$ A. Gude,${ }^{141}$ J. Haupt,${ }^{141}$ S. C. Kao, ${ }^{141}$ K. Klapoetke, ${ }^{141}$ Y. Kubota, ${ }^{141}$ J. Mans, ${ }^{141}$ N. Pastika,${ }^{141}$ R. Rusack,${ }^{141}$ M. Sasseville, ${ }^{141}$ A. Singovsky, ${ }^{141}$ N. Tambe, ${ }^{141}$ J. Turkewitz, ${ }^{141}$ L. M. Cremaldi, ${ }^{142}$ R. Kroeger, ${ }^{142}$ L. Perera, ${ }^{142}$ R. Rahmat, ${ }^{142}$ D. A. Sanders, ${ }^{142}$ D. Summers, ${ }^{142}$ E. Avdeeva, ${ }^{143}$ K. Bloom, ${ }^{143}$ S. Bose,${ }^{143}$ D. R. Claes, ${ }^{143}$ A. Dominguez, ${ }^{143}$ M. Eads, ${ }^{143}$ R. Gonzalez Suarez, ${ }^{143}$ J. Keller, ${ }^{143}$ I. Kravchenko, ${ }^{143}$ J. Lazo-Flores, ${ }^{143}$ S. Malik, ${ }^{143}$ F. Meier, ${ }^{143}$ 
G. R. Snow, ${ }^{143}$ J. Dolen, ${ }^{144}$ A. Godshalk, ${ }^{144}$ I. Iashvili, ${ }^{144}$ S. Jain, ${ }^{144}$ A. Kharchilava, ${ }^{144}$ A. Kumar, ${ }^{144}$ S. Rappoccio ${ }^{144}$ Z. Wan, ${ }^{144}$ G. Alverson, ${ }^{145}$ E. Barberis ${ }^{145}$ D. Baumgartel, ${ }^{145}$ M. Chasco,${ }^{145}$ J. Haley,${ }^{145}$ D. Nash,${ }^{145}$ T. Orimoto, ${ }^{145}$ D. Trocino, ${ }^{145}$ D. Wood,${ }^{145}$ J. Zhang, ${ }^{145}$ A. Anastassov, ${ }^{146}$ K. A. Hahn, ${ }^{146}$ A. Kubik, ${ }^{146}$ L. Lusito, ${ }^{146}$ N. Mucia, ${ }^{146}$ N. Odell, ${ }^{146}$ B. Pollack, ${ }^{146}$ A. Pozdnyakov, ${ }^{146}$ M. Schmitt, ${ }^{146}$ S. Stoynev, ${ }^{146}$ M. Velasco, ${ }^{146}$ S. Won, ${ }^{146}$ D. Berry, ${ }^{147}$ A. Brinkerhoff, ${ }^{147}$ K. M. Chan,${ }^{147}$ M. Hildreth, ${ }^{147}$ C. Jessop, ${ }^{147}$ D. J. Karmgard, ${ }^{147}$ J. Kolb, ${ }^{147}$ K. Lannon, ${ }^{147}$ W. Luo, ${ }^{147}$ S. Lynch,${ }^{147}$ N. Marinelli, ${ }^{147}$ D. M. Morse, ${ }^{147}$ T. Pearson, ${ }^{147}$ M. Planer, ${ }^{147}$ R. Ruchti, ${ }^{147}$ J. Slaunwhite, ${ }^{147}$ N. Valls, ${ }^{147}$ M. Wayne,${ }^{147}$ M. Wolf,${ }^{147}$ L. Antonelli, ${ }^{148}$ B. Bylsma, ${ }^{148}$ L. S. Durkin, ${ }^{148}$ C. Hill, ${ }^{148}$ R. Hughes, ${ }^{148}$ K. Kotov, ${ }^{148}$ T. Y. Ling, ${ }^{148}$ D. Puigh,${ }^{148}$ M. Rodenburg, ${ }^{148}$ G. Smith, ${ }^{148}$ C. Vuosalo, ${ }^{148}$ G. Williams,${ }^{148}$ B. L. Winer, ${ }^{148}$ H. Wolfe ${ }^{148}$ E. Berry, ${ }^{149}$ P. Elmer, ${ }^{149}$ V. Halyo, ${ }^{149}$ P. Hebda,${ }^{149}$ J. Hegeman, ${ }^{149}$ A. Hunt, ${ }^{149}$ P. Jindal, ${ }^{149}$ S. A. Koay, ${ }^{149}$ D. Lopes Pegna, ${ }^{149}$ P. Lujan, ${ }^{149}$ D. Marlow, ${ }^{149}$ T. Medvedeva, ${ }^{149}$ M. Mooney, ${ }^{149}$ J. Olsen, ${ }^{149}$ P. Piroué ${ }^{149}$ X. Quan, ${ }^{149}$ A. Raval, ${ }^{149}$ H. Saka, ${ }^{149}$ D. Stickland,${ }^{149}$ C. Tully, ${ }^{149}$ J. S. Werner, ${ }^{149}$ S. C. Zenz, ${ }^{149}$ A. Zuranski, ${ }^{149}$ E. Brownson, ${ }^{150}$ A. Lopez, ${ }^{150}$ H. Mendez, ${ }^{150}$ J. E. Ramirez Vargas, ${ }^{150}$ E. Alagoz, ${ }^{151}$ D. Benedetti, ${ }^{151}$ G. Bolla, ${ }^{151}$ D. Bortoletto,${ }^{151}$ M. De Mattia, ${ }^{151}$ A. Everett,${ }^{151}$ Z. Hu, ${ }^{151}$ M. Jones, ${ }^{151}$ K. Jung, ${ }^{151}$ O. Koybasi, ${ }^{151}$ M. Kress, ${ }^{151}$ N. Leonardo, ${ }^{151}$ V. Maroussov, ${ }^{151}$ P. Merkel, ${ }^{151}$ D. H. Miller,${ }^{151}$ N. Neumeister, ${ }^{151}$ I. Shipsey, ${ }^{151}$ D. Silvers, ${ }^{151}$ A. Svyatkovskiy, ${ }^{151}$ M. Vidal Marono, ${ }^{151}$ F. Wang, ${ }^{151}$ L. Xu,${ }^{151}$ H. D. Yoo, ${ }^{151}$ J. Zablocki, ${ }^{151}$ Y. Zheng, ${ }^{151}$ S. Guragain, ${ }^{152}$ N. Parashar, ${ }^{152}$ A. Adair, ${ }^{153}$ B. Akgun, ${ }^{153}$ K. M. Ecklund, ${ }^{153}$ F. J. M. Geurts, ${ }^{153}$ W. Li ${ }^{153}$ B. P. Padley, ${ }^{153}$ R. Redjimi, ${ }^{153}$ J. Roberts, ${ }^{153}$ J. Zabel, ${ }^{153}$ B. Betchart, ${ }^{154}$ A. Bodek, ${ }^{154}$ R. Covarelli, ${ }^{154}$ P. de Barbaro, ${ }^{154}$ R. Demina, ${ }^{154}$ Y. Eshaq, ${ }^{154}$ T. Ferbel, ${ }^{154}$ A. Garcia-Bellido, ${ }^{154}$ P. Goldenzweig, ${ }^{154}$ J. Han, ${ }^{154}$ A. Harel, ${ }^{154}$ D. C. Miner, ${ }^{154}$ G. Petrillo, ${ }^{154}$ D. Vishnevskiy, ${ }^{154}$ M. Zielinski, ${ }^{154}$ A. Bhatti, ${ }^{155}$ R. Ciesielski, ${ }^{155}$ L. Demortier, ${ }^{155}$ K. Goulianos, ${ }^{155}$ G. Lungu, ${ }^{155}$ S. Malik, ${ }^{155}$ C. Mesropian, ${ }^{155}$ S. Arora,${ }^{156}$ A. Barker, ${ }^{156}$ J. P. Chou, ${ }^{156}$ C. Contreras-Campana, ${ }^{156}$ E. Contreras-Campana, ${ }^{156}$ D. Duggan, ${ }^{156}$ D. Ferencek, ${ }^{156}$ Y. Gershtein, ${ }^{156}$ R. Gray, ${ }^{156}$ E. Halkiadakis, ${ }^{156}$ D. Hidas, ${ }^{156}$ A. Lath, ${ }^{156}$ S. Panwalkar, ${ }^{156}$ M. Park, ${ }^{156}$ R. Patel, ${ }^{156}$ V. Rekovic, ${ }^{156}$ J. Robles, ${ }^{156}$ K. Rose,${ }^{156}$ S. Salur, ${ }^{156}$ S. Schnetzer, ${ }^{156}$ C. Seitz, ${ }^{156}$ S. Somalwar, ${ }^{156}$ R. Stone,${ }^{156}$ S. Thomas, ${ }^{156}$ M. Walker, ${ }^{156}$ G. Cerizza ${ }^{157}$ M. Hollingsworth, ${ }^{157}$ S. Spanier, ${ }^{157}$ Z. C. Yang, ${ }^{157}$ A. York,,${ }^{157}$ R. Eusebi, ${ }^{158}$ W. Flanagan, ${ }^{158}$ J. Gilmore, ${ }^{158}$ T. Kamon, ${ }^{158, \text { iii }}$ V. Khotilovich, ${ }^{158}$ R. Montalvo, ${ }^{158}$ I. Osipenkov, ${ }^{158}$ Y. Pakhotin, ${ }^{158}$ A. Perloff, ${ }^{158}$ J. Roe,${ }^{158}$ A. Safonov, ${ }^{158}$ T. Sakuma, ${ }^{158}$ I. Suarez,${ }^{158}$ A. Tatarinov, ${ }^{158}$ D. Toback,${ }^{158}$ N. Akchurin, ${ }^{159}$ J. Damgov, ${ }^{159}$ C. Dragoiu, ${ }^{159}$ P. R. Dudero, ${ }^{159}$ C. Jeong, ${ }^{159}$ K. Kovitanggoon, ${ }^{159}$ S. W. Lee, ${ }^{159}$

T. Libeiro, ${ }^{159}$ I. Volobouev, ${ }^{159}$ E. Appelt, ${ }^{160}$ A. G. Delannoy, ${ }^{160}$ S. Greene, ${ }^{160}$ A. Gurrola, ${ }^{160}$ W. Johns, ${ }^{160}$

C. Maguire, ${ }^{160}$ Y. Mao, ${ }^{160}$ A. Melo, ${ }^{160}$ M. Sharma, ${ }^{160}$ P. Sheldon, ${ }^{160}$ B. Snook, ${ }^{160}$ S. Tuo, ${ }^{160}$ J. Velkovska, ${ }^{160}$ M. W. Arenton, ${ }^{161}$ S. Boutle, ${ }^{161}$ B. Cox,${ }^{161}$ B. Francis, ${ }^{161}$ J. Goodell, ${ }^{161}$ R. Hirosky, ${ }^{161}$ A. Ledovskoy, ${ }^{161}$ C. Lin,${ }^{161}$

C. Neu, ${ }^{161}$ J. Wood, ${ }^{161}$ S. Gollapinni, ${ }^{162}$ R. Harr, ${ }^{162}$ P. E. Karchin, ${ }^{162}$ C. Kottachchi Kankanamge Don, ${ }^{162}$ P. Lamichhane, ${ }^{162}$ A. Sakharov, ${ }^{162}$ M. Anderson, ${ }^{163}$ D. A. Belknap, ${ }^{163}$ L. Borrello, ${ }^{163}$ D. Carlsmith, ${ }^{163}$ M. Cepeda, ${ }^{163}$ S. Dasu, ${ }^{163}$ E. Friis, ${ }^{163}$ K. S. Grogg, ${ }^{163}$ M. Grothe, ${ }^{163}$ R. Hall-Wilton, ${ }^{163}$ M. Herndon, ${ }^{163}$ A. Hervé, ${ }^{163}$ K. Kaadze, ${ }^{163}$ P. Klabbers, ${ }^{163}$ J. Klukas, ${ }^{163}$ A. Lanaro, ${ }^{163}$ C. Lazaridis, ${ }^{163}$ R. Loveless, ${ }^{163}$ A. Mohapatra, ${ }^{163}$ M. U. Mozer, ${ }^{163}$ I. Ojalvo, ${ }^{163}$ G. A. Pierro, ${ }^{163}$ I. Ross, ${ }^{163}$ A. Savin,,${ }^{163}$ W. H. Smith, ${ }^{163}$ and J. Swanson ${ }^{163}$

(CMS Collaboration)

\author{
${ }^{1}$ Yerevan Physics Institute, Yerevan, Armenia \\ ${ }^{2}$ Institut für Hochenergiephysik der OeAW, Wien, Austria \\ ${ }^{3}$ National Centre for Particle and High Energy Physics, Minsk, Belarus \\ ${ }^{4}$ Universiteit Antwerpen, Antwerpen, Belgium \\ ${ }^{5}$ Vrije Universiteit Brussel, Brussel, Belgium \\ ${ }^{6}$ Université Libre de Bruxelles, Bruxelles, Belgium \\ ${ }^{7}$ Ghent University, Ghent, Belgium \\ ${ }^{8}$ Université Catholique de Louvain, Louvain-la-Neuve, Belgium \\ ${ }^{9}$ Université de Mons, Mons, Belgium \\ ${ }^{10}$ Centro Brasileiro de Pesquisas Fisicas, Rio de Janeiro, Brazil \\ ${ }^{11}$ Universidade do Estado do Rio de Janeiro, Rio de Janeiro, Brazil \\ ${ }^{12 a}$ Universidade Estadual Paulista, São Paulo, Brazil \\ ${ }^{12 \mathrm{~b}}$ Universidade Federal do ABC, São Paulo, Brazil \\ ${ }^{13}$ Institute for Nuclear Research and Nuclear Energy, Sofia, Bulgaria \\ ${ }^{14}$ University of Sofia, Sofia, Bulgaria \\ ${ }^{15}$ Institute of High Energy Physics, Beijing, China
}


${ }^{16}$ State Key Laboratory of Nuclear Physics and Technology, Peking University, Beijing, China

${ }^{17}$ Universidad de Los Andes, Bogota, Colombia

${ }^{18}$ Technical University of Split, Split, Croatia

${ }^{19}$ University of Split, Split, Croatia

${ }^{20}$ Institute Rudjer Boskovic, Zagreb, Croatia

${ }^{21}$ University of Cyprus, Nicosia, Cyprus

${ }^{22}$ Charles University, Prague, Czech Republic

${ }^{23}$ Academy of Scientific Research and Technology of the Arab Republic of Egypt,

Egyptian Network of High Energy Physics, Cairo, Egypt

${ }^{24}$ National Institute of Chemical Physics and Biophysics, Tallinn, Estonia

${ }^{25}$ Department of Physics, University of Helsinki, Helsinki, Finland

${ }^{26}$ Helsinki Institute of Physics, Helsinki, Finland

${ }^{27}$ Lappeenranta University of Technology, Lappeenranta, Finland

${ }^{28}$ DSM/IRFU, CEA/Saclay, Gif-sur-Yvette, France

${ }^{29}$ Laboratoire Leprince-Ringuet, Ecole Polytechnique, IN2P3-CNRS, Palaiseau, France

${ }^{30}$ Institut Pluridisciplinaire Hubert Curien, Université de Strasbourg, Université de Haute Alsace Mulhouse, CNRS/IN2P3, Strasbourg, France

${ }^{31}$ Centre de Calcul de l'Institut National de Physique Nucleaire et de Physique des Particules, CNRS/IN2P3, Villeurbanne, France

${ }^{32}$ Université de Lyon, Université Claude Bernard Lyon 1, CNRS-IN2P3, Institut de Physique Nucléaire de Lyon, Villeurbanne, France

${ }^{33}$ Institute of High Energy Physics and Informatization, Tbilisi State University, Tbilisi, Georgia

${ }^{34}$ RWTH Aachen University, I. Physikalisches Institut, Aachen, Germany

${ }^{35}$ RWTH Aachen University, III. Physikalisches Institut A, Aachen, Germany

${ }^{36}$ RWTH Aachen University, III. Physikalisches Institut B, Aachen, Germany

${ }^{37}$ Deutsches Elektronen-Synchrotron, Hamburg, Germany

${ }^{38}$ University of Hamburg, Hamburg, Germany

${ }^{39}$ Institut für Experimentelle Kernphysik, Karlsruhe, Germany

${ }^{40}$ Institute of Nuclear and Particle Physics (INPP), NCSR Demokritos, Aghia Paraskevi, Greece

${ }^{41}$ University of Athens, Athens, Greece

${ }^{42}$ University of Ioánnina, Ioánnina, Greece

${ }^{43}$ KFKI Research Institute for Particle and Nuclear Physics, Budapest, Hungary

${ }^{44}$ Institute of Nuclear Research ATOMKI, Debrecen, Hungary

${ }^{45}$ University of Debrecen, Debrecen, Hungary

${ }^{46}$ Panjab University, Chandigarh, India

${ }^{47}$ University of Delhi, Delhi, India

${ }^{48}$ Saha Institute of Nuclear Physics, Kolkata, India

${ }^{49}$ Bhabha Atomic Research Centre, Mumbai, India

${ }^{50}$ Tata Institute of Fundamental Research-EHEP, Mumbai, India

${ }^{51}$ Tata Institute of Fundamental Research-HECR, Mumbai, India

${ }^{52}$ Institute for Research in Fundamental Sciences (IPM), Tehran, Iran

${ }^{53}$ University College Dublin, Dublin, Ireland

${ }^{54 a}$ INFN Sezione di Bari, Bari, Italy

${ }^{54 \mathrm{~b}}$ Università di Bari, Bari, Italy

${ }^{54 \mathrm{c}}$ Politecnico di Bari, Bari, Italy

${ }^{55}$ INFN Sezione di Bologna, Bologna, Italy

${ }^{55 \mathrm{~b}}$ Università di Bologna, Bologna, Italy

${ }^{56 a}$ INFN Sezione di Catania, Catania, Italy

${ }^{56 \mathrm{~b}}$ Università di Catania, Catania, Italy

${ }^{57}$ INFN Sezione di Firenze, Firenze, Italy

${ }^{57 b}$ Università di Firenze, Firenze, Italy

${ }^{58}$ INFN Laboratori Nazionali di Frascati, Frascati, Italy

${ }^{59 a}$ INFN Sezione di Genova, Genova, Italy

${ }^{59 \mathrm{~b}}$ Università di Genova, Genova, Italy

${ }^{60 \mathrm{a}}$ INFN Sezione di Milano-Bicocca, Milano, Italy

${ }^{60 \mathrm{~b}}$ Università di Milano-Bicocca, Milano, Italy

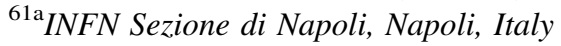

${ }^{61 \mathrm{~b}}$ Università di Napoli 'Federico II', Napoli, Italy

${ }^{61 \mathrm{c}}$ Università della Basilicata (Potenza), Napoli, Italy

${ }^{61 \mathrm{~d}}$ Università G. Marconi (Roma), Napoli, Italy

${ }^{62 \mathrm{a}}$ INFN Sezione di Padova, Padova, Italy

${ }^{62 \mathrm{~b}}$ Università di Padova, Padova, Italy

${ }^{62 \mathrm{c}}$ Università di Trento (Trento), Padova, Italy 
${ }^{63 a}$ INFN Sezione di Pavia, Pavia, Italy

${ }^{63 \mathrm{~b}}$ Università di Pavia, Pavia, Italy

${ }^{64 a}$ INFN Sezione di Perugia, Perugia, Italy

${ }^{64 \mathrm{~b}}$ Università di Perugia, Perugia, Italy

${ }^{65 a}$ INFN Sezione di Pisa, Pisa, Italy

${ }^{65 \mathrm{~b}}$ Università di Pisa, Pisa, Italy

${ }^{65 \mathrm{c}}$ Scuola Normale Superiore di Pisa, Pisa, Italy

${ }^{66 a}$ INFN Sezione di Roma, Roma, Italy

${ }^{66 \mathrm{~b}}$ Università di Roma, Roma, Italy

${ }^{67 a}$ INFN Sezione di Torino, Torino, Italy

${ }^{67 \mathrm{~b}}$ Università di Torino, Torino, Italy

${ }^{67 \mathrm{c}}$ Università del Piemonte Orientale (Novara), Torino, Italy

${ }^{68 \mathrm{a}}$ INFN Sezione di Trieste, Trieste, Italy

${ }^{68 \mathrm{~b}}$ Università di Trieste, Trieste, Italy

${ }^{69}$ Kangwon National University, Chunchon, Korea

${ }^{70}$ Kyungpook National University, Daegu, Korea

${ }^{71}$ Chonnam National University, Institute for Universe and Elementary Particles, Kwangju, Korea

${ }^{72}$ Korea University, Seoul, Korea

${ }^{73}$ University of Seoul, Seoul, Korea

${ }^{74}$ Sungkyunkwan University, Suwon, Korea

${ }^{75}$ Vilnius University, Vilnius, Lithuania

${ }^{76}$ Centro de Investigacion y de Estudios Avanzados del IPN, Mexico City, Mexico

${ }^{77}$ Universidad Iberoamericana, Mexico City, Mexico

${ }^{78}$ Benemerita Universidad Autonoma de Puebla, Puebla, Mexico

${ }^{79}$ Universidad Autónoma de San Luis Potosí, San Luis Potosí, Mexico

${ }^{80}$ University of Auckland, Auckland, New Zealand

${ }^{81}$ University of Canterbury, Christchurch, New Zealand

${ }^{82}$ National Centre for Physics, Quaid-I-Azam University, Islamabad, Pakistan

${ }^{83}$ National Centre for Nuclear Research, Swierk, Poland

${ }^{84}$ Institute of Experimental Physics, Faculty of Physics, University of Warsaw, Warsaw, Poland

${ }^{85}$ Laboratório de Instrumentação e Física Experimental de Partículas, Lisboa, Portugal

${ }^{86}$ Joint Institute for Nuclear Research, Dubna, Russia

${ }^{87}$ Petersburg Nuclear Physics Institute, Gatchina (St. Petersburg), Russia

${ }^{88}$ Institute for Nuclear Research, Moscow, Russia

${ }^{89}$ Institute for Theoretical and Experimental Physics, Moscow, Russia

${ }^{90}$ P.N. Lebedev Physical Institute, Moscow, Russia

${ }^{91}$ Skobeltsyn Institute of Nuclear Physics, Lomonosov Moscow State University, Moscow, Russia

${ }^{92}$ State Research Center of Russian Federation, Institute for High Energy Physics, Protvino, Russia

${ }^{93}$ University of Belgrade, Faculty of Physics and Vinca Institute of Nuclear Sciences, Belgrade, Serbia

${ }^{94}$ Centro de Investigaciones Energéticas Medioambientales y Tecnológicas (CIEMAT), Madrid, Spain

${ }^{95}$ Universidad Autónoma de Madrid, Madrid, Spain

${ }^{96}$ Universidad de Oviedo, Oviedo, Spain

${ }^{97}$ Instituto de Física de Cantabria (IFCA), CSIC-Universidad de Cantabria, Santander, Spain

${ }^{98}$ CERN, European Organization for Nuclear Research, Geneva, Switzerland

${ }^{99}$ Paul Scherrer Institut, Villigen, Switzerland

${ }^{100}$ Institute for Particle Physics, ETH Zurich, Zurich, Switzerland

${ }^{101}$ Universität Zürich, Zurich, Switzerland

${ }^{102}$ National Central University, Chung-Li, Taiwan

${ }^{103}$ National Taiwan University (NTU), Taipei, Taiwan

${ }^{104}$ Chulalongkorn University, Bangkok, Thailand

${ }^{105}$ Cukurova University, Adana, Turkey

${ }^{106}$ Middle East Technical University, Physics Department, Ankara, Turkey

${ }^{107}$ Bogazici University, Istanbul, Turkey

${ }^{108}$ Istanbul Technical University, Istanbul, Turkey

${ }^{109}$ National Scientific Center, Kharkov Institute of Physics and Technology, Kharkov, Ukraine

${ }^{110}$ University of Bristol, Bristol, United Kingdom

${ }^{111}$ Rutherford Appleton Laboratory, Didcot, United Kingdom

${ }^{112}$ Imperial College, London, United Kingdom

${ }^{113}$ Brunel University, Uxbridge, United Kingdom

${ }^{114}$ Baylor University, Waco, Texas, USA

${ }^{115}$ The University of Alabama, Tuscaloosa, Alabama, USA 


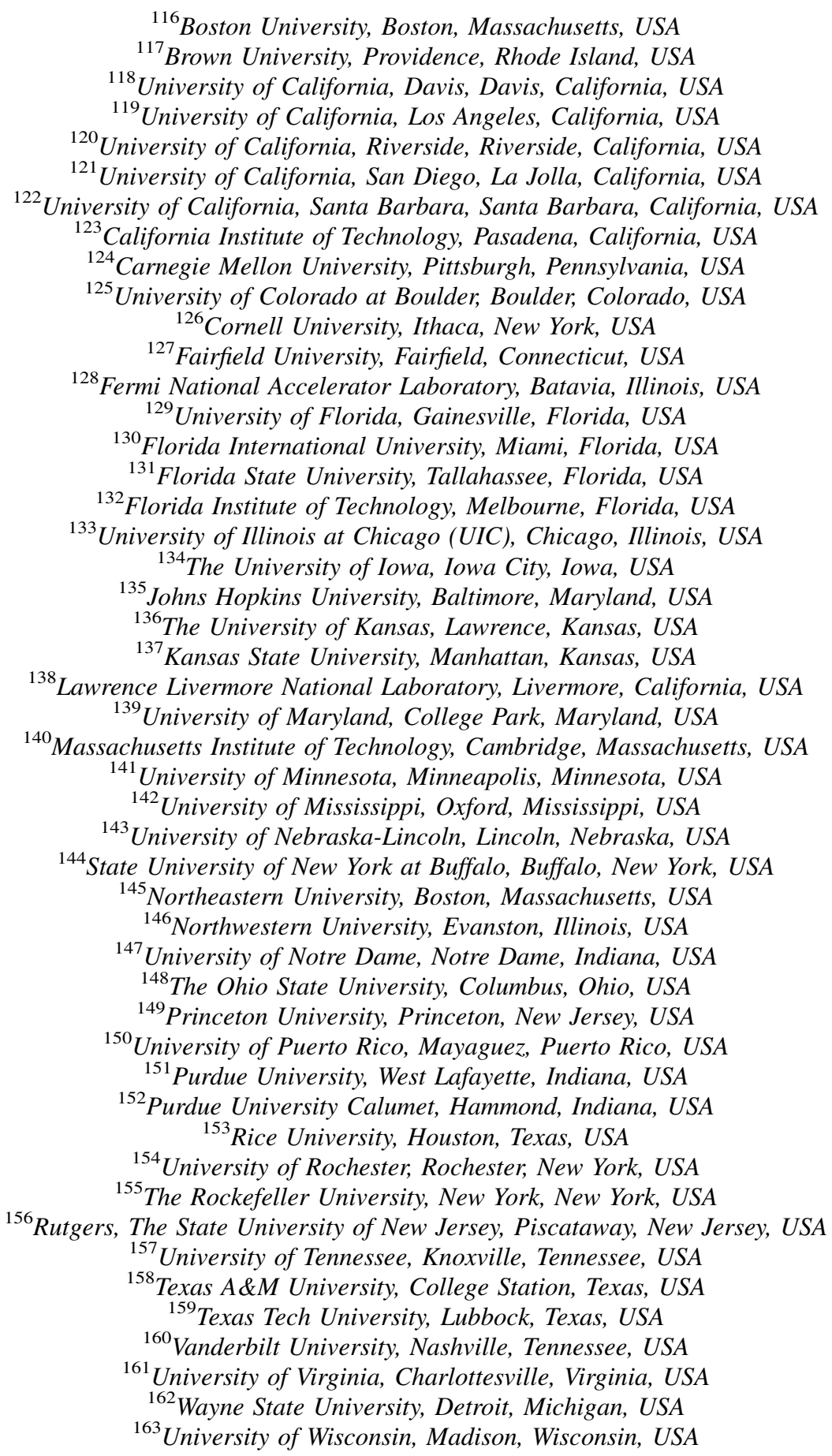

${ }^{\mathrm{a}}$ Deceased.

${ }^{\mathrm{b}}$ Also at Vienna University of Technology, Vienna, Austria.

${ }^{\mathrm{c}}$ Also at CERN, European Organization for Nuclear Research, Geneva, Switzerland.

${ }^{\mathrm{d}}$ Also at Institut Pluridisciplinaire Hubert Curien, Université de Strasbourg, Université de Haute Alsace Mulhouse, CNRS/IN2P3, Strasbourg, France.

${ }^{\mathrm{e}}$ Also at National Institute of Chemical Physics and Biophysics, Tallinn, Estonia.

${ }^{\mathrm{f}}$ Also at Skobeltsyn Institute of Nuclear Physics, Lomonosov Moscow State University, Moscow, Russia.

${ }^{\mathrm{g}}$ Also at Universidade Estadual de Campinas, Campinas, Brazil.

${ }^{\mathrm{h}}$ Also at California Institute of Technology, Pasadena, CA, USA.

${ }^{\mathrm{i}}$ Also at Laboratoire Leprince-Ringuet, Ecole Polytechnique, IN2P3-CNRS, Palaiseau, France.

${ }^{j}$ Also at Suez Canal University, Suez, Egypt. 
${ }^{\mathrm{k}}$ Also at Cairo University, Cairo, Egypt.

${ }^{1}$ Also at Fayoum University, El-Fayoum, Egypt.

${ }^{\mathrm{m}}$ Also at Helwan University, Cairo, Egypt.

${ }^{\mathrm{n}}$ Also at British University in Egypt, Cairo, Egypt.

${ }^{\circ}$ Now at Ain Shams University, Cairo, Egypt.

${ }^{\mathrm{p}}$ Also at National Centre for Nuclear Research, Swierk, Poland.

${ }^{\mathrm{q}}$ Also at Université de Haute Alsace, Mulhouse, France.

${ }^{\mathrm{r}}$ Also at Joint Institute for Nuclear Research, Dubna, Russia.

sAlso at Brandenburg University of Technology, Cottbus, Germany.

${ }^{\mathrm{t}}$ Also at The University of Kansas, Lawrence, KS, USA.

${ }^{\mathrm{u}}$ Also at Institute of Nuclear Research ATOMKI, Debrecen, Hungary.

${ }^{v}$ Also at Eötvös Loránd University, Budapest, Hungary.

${ }^{\mathrm{w}}$ Also at Tata Institute of Fundamental Research-HECR, Mumbai, India.

${ }^{\mathrm{x}}$ Now at King Abdulaziz University, Jeddah, Saudi Arabia.

${ }^{y}$ Also at University of Visva-Bharati, Santiniketan, India.

${ }^{\mathrm{z}}$ Also at University of Ruhuna, Matara, Sri Lanka.

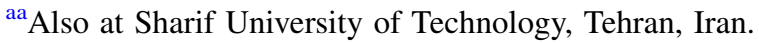

${ }^{\mathrm{bb}}$ Also at Isfahan University of Technology, Isfahan, Iran.

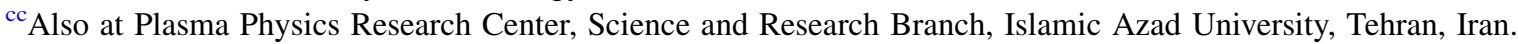

${ }^{\mathrm{dd}}$ Also at Università degli Studi di Siena, Siena, Italy.

${ }^{e e}$ Also at Universidad Michoacana de San Nicolas de Hidalgo, Morelia, Mexico.

${ }^{\mathrm{ff}}$ Also at Faculty of Physics, University of Belgrade, Belgrade, Serbia.

${ }^{\mathrm{gg}}$ Also at Facoltà Ingegneria, Università di Roma, Roma, Italy.

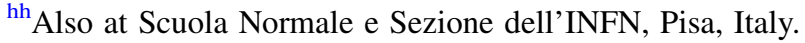

${ }^{i i}$ Also at INFN Sezione di Roma, Roma, Italy.

${ }^{\mathrm{jj}}$ Also at University of Athens, Athens, Greece.

${ }^{\mathrm{kk}}$ Also at Rutherford Appleton Laboratory, Didcot, United Kingdom.

${ }^{11}$ Also at Paul Scherrer Institut, Villigen, Switzerland.

${ }^{\mathrm{mm}}$ Also at Institute for Theoretical and Experimental Physics, Moscow, Russia.

${ }^{\mathrm{nn}}$ Also at Albert Einstein Center for Fundamental Physics, Bern, Switzerland.

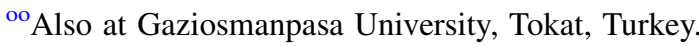

${ }^{\mathrm{pp} A l s o}$ at Adiyaman University, Adiyaman, Turkey.

${ }^{\mathrm{qq}} \mathrm{Also}$ at Cag University, Mersin, Turkey.

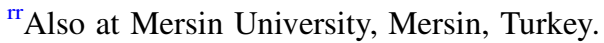

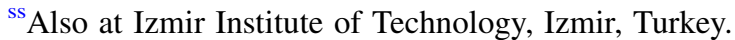

${ }^{t t}$ Also at Ozyegin University, Istanbul, Turkey.

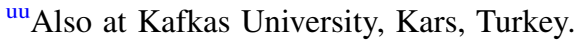

${ }^{\mathrm{vv}}$ Also at Suleyman Demirel University, Isparta, Turkey.

${ }^{\text {ww }}$ Also at Ege University, Izmir, Turkey.

${ }^{\mathrm{xx}}$ Also at Mimar Sinan University, Istanbul, Istanbul, Turkey.

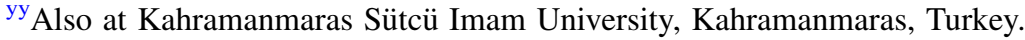

${ }^{\mathrm{zz}}$ Also at School of Physics and Astronomy, University of Southampton, Southampton, United Kingdom.

${ }^{\text {aaa }}$ Also at INFN Sezione di Perugia, Università di Perugia, Perugia, Italy.

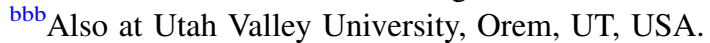

${ }^{\mathrm{ccc}}$ Also at University of Edinburgh, Scotland, Edinburgh, United Kingdom.

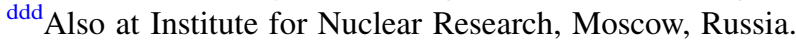

${ }^{e e e}$ Also at University of Belgrade, Faculty of Physics and Vinca Institute of Nuclear Sciences, Belgrade, Serbia.

${ }^{f f f}$ Also at Argonne National Laboratory, Argonne, IL, USA.

${ }^{\text {ggg }}$ Also at Erzincan University, Erzincan, Turkey.

${ }^{\text {hhh }}$ Also at Yildiz Technical University, Istanbul, Turkey.

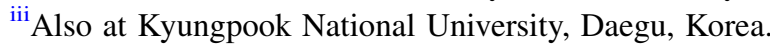

